A N N A L E S Annales de Bretagne et des Pays de l'Ouest

Anjou. Maine. Poitou-Charente. Touraine

$113-2 \mid 2006$

Varia

\title{
La recherche à l'université de Nantes de 1998 à 2005
}

\section{(2) OpenEdition}

Journals

Édition électronique

URL : http://journals.openedition.org/abpo/886

DOI : $10.4000 / a b p o .886$

ISBN : 978-2-7535-1502-4

ISSN : 2108-6443

Éditeur

Presses universitaires de Rennes

Édition imprimée

Date de publication : 30 juin 2006

Pagination : 219-250

ISBN : 978-2-7535-0331-1

ISSN : $0399-0826$

\section{Référence électronique}

"La recherche à l'université de Nantes de 1998 à 2005 », Annales de Bretagne et des Pays de l'Ouest [En ligne], 113-2 | 2006, mis en ligne le 30 juin 2008, consulté le 19 avril 2019. URL : http:// journals.openedition.org/abpo/886 ; DOI : 10.4000/abpo.886 


\section{La recherche à l'université de Nantes de 1998 à 2005}

Thèses

\section{5}

\section{Histoire moderne}

KouAme, Aka, Les cargaisons de traite nantaises au XVIII siècle, dir. C. HERMANN.

NouAILle-Degorce, Patrick, Zouaves pontificaux et volontaires de l'Ouest dans la Guerre de 1870-1871, dir. J.-P. BoIs.

\section{Histoire contemporaine}

EL TURC, Joëlle, La vie constitutionnelle en France et au Liban de 1926 à 1940. Étude comparée heuristique, 2005, dir. C. HERMANN.

ÉTENDARD, Anne-Valérie, Étude de la notion de transnationalisme au travers de l'œuvre et de la vie de Franz Stock, dir. M. LAUNAY.

\section{4}

\section{Histoire moderne}

BOUYER, Murielle, Les gens de mer de la Rivière de Loire : étude d'un bassin de main-d'œuvre du commerce nantais au XVIII siècle, dir. G. SAUPIN.

PICAUD, Sandrine, La petite guerre au XVIII siècle : l'exemple des campagnes de Flandre de la guerre de Succession d'Autriche, mises en perspective dans la pensée française et européenne, dir. J.P. BoIs.

\section{Histoire contemporaine}

La Foye MElEard, Sylvie de, Les répercussions du conflit israélo-arabe sur les relations américano-égyptiennes de novembre 1968 à août 1974, dir. Y.-H. NOUAILHAT.

Poncelet, Yves, La méthode de l'abstraction extensive : des événements aux objets dans la philosophie de la nature d'Alfred North Whitehead, dir. M. Launay.

\section{3}

\section{Histoire ancienne}

HugOT, Laurent, Recherches sur le sacrifice en Étrurie, dir. J.-R. JANNOT.

\section{Histoire médiévale}

CHOTARD, Stéphanie, Les eaux et forêts du bailliage de Chalon aux XIV et XV siècles, dir. J.-L. SARRAZIN.

\section{Histoire moderne}

PERREON, Stéphane, L'armée en Bretagne au XVIII e siècle 1689-1789, dir. J.-P. BoIs.

\section{Histoire contemporaine}

GAUTIER, Marie-José, L'évolution politique du Sud Soudan de 1956 à 1989, dir. J. WEBER.

\section{2}

\section{Archéologie}

MENs, Emmanuel, Approche technologique de l'art gravé néolithique d'Europe occidentale : de l'extraction des supports aux techniques d'exécutions des gravures, dir. S. CASSEN.

ARs, François, La société polymathique du Morbihan et la conservation du patrimoine mégalithique, dir. M. LAUNAY.

\section{Histoire ancienne}

OuACHOUR, Fatima, Le métissage culturel en Afrique du Nord ancienne : approches conceptuelles, dir. J. PEYRAS. 


\section{Histoire contemporaine}

BenSACQ-TeXIEr, Nicole, Le corps diplomatique français en Chine (1840-1911). Origines, formation, statut, organisation, fonctions, dir. (J.) WEBER.

BERTHET, Samuel, L'action culturelle de la France en Inde de 1914 à 1985, dir. J. WEBER.

Kолок, Salma, L'immigration libanaise en Côte-d'Ivoire (1920-1960), dir. M. LAUNAY.

\section{1}

\section{Archéologie}

HERBAUT, Frédéric, La parure néolithique dans l'Ouest de la France, dir. S. CASSEN.

\section{Histoire contemporaine}

BERNARD, Yveline, Les patronages catholiques dans le diocèse de Nantes de leur origine jusqu'à la mission de 1958, dir. M. LAUNAY.

DeshaYes, Laurent, La mission du Tibet 1846-1952. Des pionniers, des prêtres, des Français entre Chine et Tibet, dir. J. WEBER.

Holveque-Pain, Stéphanie, La politique de la communauté européenne à l'égard des pays de l'Europe de l'Est : dialogue, désaccord, coopération (1969-1991), dir. J. WEBER.

VASQUEZ, Manuel, La route de la feuille de coca 1492-1992. Entre l'histoire, la mémoire et l'oubli, dir. J.-C. MARTIN.

\section{0}

\section{Histoire ancienne}

PIEL, Thierry, Recherches sur les représentations et la nature du pouvoir en Etrurie $\left(\mathrm{VII}^{\mathrm{e}} \mathrm{I}^{\mathrm{er}}\right.$ siècles avant J.-C.), dir. J.-R. JANNOT.

Sichet, Sandra, La magie dans l'Afrique romaine, dir. J. PEYRAS.

\section{Histoire médiévale}

GuILlaume, Gwenaël, Les Rieux, rôle politique, diplomatique, économique et militaire en Bretagne aux XIV et $X V^{e}$ siècles, dir. M. LE MENE.
JosSERAND, Philippe, Église et pouvoir dans la péninsule ibérique : les ordres militaires dans le royaume de Castille, dir. R. DURAND.

\section{Histoire contemporaine}

ElA, Emmanuel, La politique de défense du Cameroun depuis 1959: contraintes et réalités, dir. Y.-H. NOUAILHAT.

\section{9}

\section{Histoire contemporaine}

Bitoung-Angome, Michelle, Le système diplomatique gabonais en Afrique équatoriale, dir. J.-C. AlLAIN.

Boulestreau-Rolland, Anne, Sociabilités, pouvoirs et notabilités en Anjou, 17301830. Trois communautés des Mauges à l'épreuve de la guerre, dir. J.-C. MARTIN.

DENIE, Laurence, La France dans l'opinion publique américaine 1981-1988, dir. Y.-H. Nouallhat.

DouCET, Fabienne, La marine française en Indochine durant la guerre 19451954: coopération avec l'armée de terre, dir. Y.-H. NouAILHAT.

LE GARS, Gaëlle, Les relations entre les États-Unis et Tä̈wan de 1972 à 1996 : La contribution américaine à l'identité internationale de Taiwan, dir. Y.-H. NOUAILHAT.

VERBAERE, Laure, Le nietzscheisme français. Approche historique de la réception de Nietzsche en France de 1872 à 1910, dir. J.-C. MARTIN.

\section{8}

\section{Histoire médiévale}

LE FRAPPER-LeRICHE, Reine, La justice en Bretagne à la fin du Moyen Âge, dir. M. LE MENE.

\section{Histoire moderne}

RoGER, Paul, Les marines de guerre européennes vues par les peintres et graveurs, $X V I^{e}-X I X^{e}$ siècles, dir. J.-P. BoIs. 


\section{Histoire contemporaine}

BEL IL, Dominique, La réception du concile de Vatican II par les médias dans le diocèse de Nantes, dir. M. LaUnAY.

DENECHERE, Yves, La politique espagnole de la France de 1931 à 1936, dir. Y.-H. NOUAILHAT.

Le Pautremat, Pascal, Le rôle de la Commission interministérielle des affaires musulmanes dans l'élaboration d'une politique musulmane de la France de 1911 à 1938, dir. Y.-H. NouAlLHAT.

MisRa-BESNARD, Kamakshi, Le nationalisme en Inde : le cas de Chandernagor, le premier maillon de la chaîne, 1905-1951, dir. J. WEBER.

NzENZE, Mireille, L'armée française en Afrique équatoriale française de 1919 à 1958, dir. J.-C. ALLAIN.

\section{Diplômes d'Études Approfondies (DEA) - Master 2}

\section{5}

\section{Histoire médiévale}

GAUTHEY, Thomas, L'éléphant : un grand animal exotique dans l'Occident médiéval, dir. J. TOLAN.

\section{Histoire contemporaine}

ARAUJo, Frédéric, L'Orientalisme au XIXe siècle : l'exemple des peintures du Musée des Beaux Arts de Nantes, dir. R. FABRE.

BERNARD, Jérôme, La crise ivoirienne d'août 1999 à novembre 2004 vue par la presse burkinabée (Sidwaya, Journal du Jeudi), dir. R. FABRE.

BoISDRON, Matthieu, À la recherche d'une impossible sécurité collective pour l'Europe centrale et orientale dans les années Trente, dir. B. JoLY.

CAREY, Ciaran, La France et « la question irlandaise "1914-1923 : la guerre d'Indépendance et la guerre civile en Irlande vues par la diplomatie française, dir. M. Catala.

Edzegue-Mendame, Aristique, La coopération Union européenne/Gabon à travers la convention de Lomé IV (19891999), dir. M. CATALA.
GALLARD, Claire, Historique des collections océaniennes du Musée départemental Dobrée, dir. J. WeBER.

GAY, Sébastien, La sécurité publique, un enjeu majeur dans la gestion des crises : forces de sécurité intérieure et opérations extérieures, dir. F. Bozo.

RAINGEARD, Pierre, Les missions intérieures en Vendée $X I X^{e}-X X^{e}$ siècles, dir. R. FABRE.

Rousse, Cédric, La société et la culture française à travers l'Ideal de Grenada aux débuts de la Ve République (juin 1958-juin 1962), dir. J. WEBER.

Schugt, Alexander G., OTAN de questions : le nucléaire et les relations transatlantiques 1977-1981, dir. F. Bozo.

\section{4}

\section{Histoire médiévale}

MINIER, Denis, L'administration d'un patrimoine aristocratique : l'exemple de La Trémoille de la fin du XIVe au début du $X V t^{e}$ siècle, dir. J.-L. SARRAZIN.

GuIot, Laurence, Une œuvre apologétique et polémique : la disputa de $\mathrm{Fez}$, dir. J. TOLAN.

\section{Histoire moderne}

BOUTERA, David, Présence et circulation des Bohémiens en Bretagne entre la fin du XV siècle et la fin du XIXe siècle, dir. G. SAupIN.

DANET, Vincent, Le " second peuple " de Nantes (1667-1792), dir. G. SAUPIN.

LEBRAT, Soizic, Sociabilités musicales au XIX $X^{e}$ siècle (1770-1914) départements de Nantes et de Vendée, dir. G. SAupin.

MERLET Jean, Les archives notariales des Sables-d'Olonne de 1650 à 1750, dir. G. SAUPIN.

PaCaud, Yves, La compétence civile du présidial de Nantes au XVIII ${ }^{e}$ siècle, dir. G. SAUPIN.

SEGRETIN, Franck, Un régiment dans une ville : le 93e R.I. à la Roche-sur-Yon (1873-1914), dir. J.-P. BoIs.

\section{Histoire contemporaine}

BARON, Hervé, La propagande chinoise anti-chrétienne à la fin de la dynastie Qing d'après les placards pamphlets 
et libellés conservés dans les archives françaises (1840-1920), dir. J. WEBER.

Costes, Patrick, Les conséquences des attentats terroristes du 11 septembre 2001 pour les services de renseignement occidentaux, dir. F. Bozo.

DEsCAMPS, Fabrice, L'application de l'Alliance franco-russe et la question d'Orient (1894-1914), dir. B. Joly.

LASSALLE, Géraldine, La politique de promotion des femmes en Inde (19741996), dir. J. WEBER.

Roland, Nicolas de, Les conceptions de l'Europe de la Défense depuis la crise irakienne de 2003, dir. F. Bozo.

SAUGER, Mélinda, Les relations franco-italiennes : la politique étrangère italienne vue par la diplomatie française (19541958), dir. F. Bozo.

Soloch, Krzysztof, La Réforme de la Bundeswehr, dir. F. Bozo.

\section{3}

\section{Histoire moderne}

PAIREAU, Valérie, Nouvelle France et guerre de Sucession d'Espagne 1702-1713: politique, stratégie et diplomatie francoanglaise autour d'une question coloniale, dir. J.-P. BoIs.

RochetEAu, Aurélien, Le réseau consulaire français aux États-Unis (17791815) : l'institution comme interface entre deux peuples, deux cultures, deux États, dir. J.-P. BoIs.

\section{Histoire contemporaine}

BARANGER, Aurélie, La perception française du rôle britannique sur la scène internationale du sommet de Saint Malo à la crise irakienne, dir. F. Bozo.

CHAPAYS, Éric, La mise en oeuvre du Concile Vatican II dans le diocèse de Nantes sous l'épiscopat de Monseigneur Vial (1966-1982), dir. M. LAUNAY.

Falabregues-Maginot, Agnès, Opinions, polémiques et réflexions, le débat atlantique français autour de l'OTAN de 1981 à 2001, dir. F. Bozo.

GeRGAUd, Mathieu, Le lycée Jules Ferry d'Antananarivo (1924-1972) : outil colo- nial ou pépinière d'élites, dir. A. TIREFORT.

LE CAM, Éric, La prolifération des armes nucléaires et balistiques en Corée du Nord (1993-2003), dir. F. Bozo.

Penot, Pierre-Étienne, La Pologne et la naissance de Solidarité (juillet-août 1980) à travers la presse française, dir. J. WEBER.

Platov, Christine, Visions russes de la nouvelle donne internationale après les attentats du 11 septembre 2001, dir. F. Bozo.

REMAUD, Jeoffrey-Gaylord, Le rôle de la France dans la chute de l'Afrique Orientale Italienne : du ministère Mandel (10 avril 1938) à la capitulation de Gondar (28 novembre 1941), dir. A. TIREFORT.

Villette-Simon, Anne-Sophie, École lä̈que et guerre scolaire. Application des lois de Jules Ferry en Vendée 1880-1918, dir. M. LAUNAY.

\section{2}

\section{Histoire médiévale}

TuRPAUd, Baptiste, Les structures seigneuriales dans le Pays de la Jou à la fin du Moyen Âge, dir. J.-L. SARRAZIN.

\section{Histoire moderne}

CHAUvire, Frédéric, La charge de cavalerie de Bayard à Seydlitz, dir. J.-P. Bois.

BITEAU, Jérôme, Le colonel François-Leon Boscal de reals, comte de Mornac (17831858), dir. J.-P. Bois.

JuHEL, Nolwenn, La réforme catholique dans le diocèse de Nantes XVII ${ }^{e}$-XVIII siècles, dir. G. SAUPIN.

Mesnier, Paul, Les diplomates dans la guerre turque de 1736-1739, dir. J.P. BoIs.

MOREAU, Laure, La diffusion de la mode en Province de 1750-1820. Rennes, Nantes et Angers. Une étude sociologique, économique, iconographique $d u$ vêtement, dir. G. SAUPIN.

Piffeteau, Sébastien, La politique française en Roumanie du traité de Paris au traité de Berlin (1856-1878) : "le grand jeu roumain ", dir. J.-P. Bors. 


\section{Histoire contemporaine}

BARBIER, Hervé, La Division Navale d'Extrême-Orient 1870-1940, dir. J. WEBER.

BARDOUX, Pierre, Au cour de la crise des Grands-Lacs. L'ancrage de la diaspora rebelle Hutu, à travers l'exemple de la Communauté rwandaise de Nairobi 1994-2002, dir. A. TIREFORT.

BASSOMPIERRE, Karène, Le métissage au Kenya de 1930 à nos jours : mémoires de métis, dir. A. TIREFORT.

BouRHIS, Michèle, La France et les missions étrangères de Paris dans les provinces du Kouang-Tong et du KouangSi 1848-1949, dir. J. WEBER.

CARRILlon, Jean-François, Narcotrafic et guerillas en Colombie (1978-1998), dir. C. HERMANN.

Challlou, Virginie, Sources et méthodes pour une histoire des engagés africains à la Réunion: de la veille de l'abolition de l'esclavage à la fin du XIXe siècle, dir. J. WeBER.

ChATELLIER, Maxime, Les aspects économiques des relations Union européenneRépublique populaire de Chine, de 1979 à 2001, dir. J. WEBER.

DuPONT, Olivier, Deux regards tournés vers un même objectif. Les relations franco-britanniques en matière de défense à la fin du $x X^{e}$ siècle. Lexemple des guerres en ex-Yougoslavie (19911995), dir. F. Bozo.

ICRE-ROBERT, Arlette, La politique européenne en matière de sensibilisation des élèves à l'Europe : son application dans la Région des Pays de la Loire, dir. M. LAUNAY.

LAMOURET, Cyril, Un président égyptien controversé : les réactions internationales à l'assassinat d'Anour el Sadate le 6 octobre 1981, dir. J. WEBER.

LE GRand DE LA Chanonie, Catherine, Les relations franco-italiennes en Tunisie 1881-1896, dir. J. WEBER.

MEHRZAD, John, Les réactions françaises concernant la révolution iranienne. Un mémoire fondé sur la presse française, dir. J. WEBER.
PERRIN, Frédérique, Les colonies racontées aux enfants : les représentations coloniales dans la littérature enfantine sous la III République (1870-1940), dir. A. TIREFORT.

PRIOUX, Matthieu, La coopération interrégionale dans l'espace atlantique : à la lumière du cas des Pays de la Loire, dir. M. LAUNAY.

RouAud, Sébastien, Les missionnaires de la société des Missions étrangères de Paris au Japon, de 1846 à 1914, dir. J. WEBER.

\section{1}

\section{Histoire ancienne}

THEBAUD, Sébastien, Les ensembles céramiques précoces de Ratiatum (Rezé, Loire-Atlantique), dir. J. PEYRAS.

\section{Histoire moderne}

Agudelo, Lucy, L'historiographie des Jésuites en Colombie, dir. C. HERMANN.

DuBors, Mathieu, Les relations nobiliaires en Bretagne au XVI siècle, d'après les lettres de rémission, dir. M. NASSIET.

Dugast, Romain, Capitaines et gouverneurs : l'État-major en Bretagne au XVI siècle (1499-1598), dir. M. NASSIET.

\section{Histoire contemporaine}

ALLARD, Mathieu, La soviétisation de l'Europe centrale et orientale vue par les diplomates français de 1943 à 1953, dir. F. Bozo.

BONNEAU, Hélène, Les relations indo-communautaires, de 1973 à nos jours, dir. J. WEBER.

ESNAULT, Matthieu, Les relations francobritanniques au Levant de 1918 à 1946, dir. J. WEBER.

FOUQUET, Stéphane, Les relations francoaméricaines en ex-Yougoslavie (19891995), dir. F. Bozo.

GIRAUD, Ronan, La médecine à Madagascar, 1896-1946. Les détours d'une science dans un contexte colonial, dir. A. TIREFORT.

GRANDHOMme, Hélène, La politique musulmane de la France au Sénégal de 1936 à 1964, dir. A. TIREFORT. 
GUIBERT, Isabelle, Les régions albanaises de Macédoine de la fin $d u X I X X^{e}$ siècle jusqu'à la première guerre balkanique, d'après les Archives diplomatiques françaises, dir. F. Bozo.

POUPARD, David, Le château de Nantes confronté au regard des habitants sous l'Ancien Régime, dir. G. SAUPIN.

\section{0}

\section{Histoire ancienne}

BiLlon, Séverine, Les rapports entre architecture et symbolisme dans les basiliques chrétiennes. Exemple de SainteSophie de Constantinople, dir. J. Peyras.

Guichaoua, Mickaël, La nature des barbares dans l'Empire romain entre Andrinople et le sac de Rome, dir. J. Peyras.

GuITTON, David, Recherches sur la fondation de Ratiatum : (Rezé, Loire Atlantique) les ensembles sigillés précoces, dir. J. PEYRAS.

MatRAs-DesGre, Marie-Laetitia, Les politiques d'armement dans le monde grec de Denys l'Ancien à Philippe V de Macédoine, dir. J. PEYRAS.

\section{Histoire médiévale}

SALAUN, Gildas, Les monnaies de la guerre de Succession de Bretagne (1341-1365), inventaire analytique des collections du Musée Dobrée à Nantes, dir. J.-L. SARRAZIN.

\section{Histoire moderne}

Chaponet, Élisabeth, Présentation des archives du musée du Sous-officier de l'École nationale des sous-officiers d'active, dir. J.-P. Bors.

CHOTARD, Pierre, Représentations, enjeux, et imaginaires des exécutions de justice criminelle : l'échafaud à Nantes aux XVII et XVIII $I^{e}$ siècles, dir. G. SAUPIN.

DuFouRnaud, Nicole, Les femmes en Bretagne au $\mathrm{XVI}^{e}$ siècle : étude des pratiques sociales et économiques. Perspectives de recherche et méthodologie, dir. M. NASSIET.

Michon, Bernard, Les relations entre Nantes et ses avant-ports aux XVII et XVIII siècles, dir. G. SAUPIN.

NouAille-Degorce, Patrick, Matricule de la légion des Volontaires de l'Ouest (Zouaves Pontificaux), dir. J.-P. BoIs.

NouAILle-Degorce, Patrick, Zouaves pontificaux et Volontaires de l'Ouest dans la guerre de 1870-1871. Présentation des Matricules régimentaires, dir. J.-P. Bors. ROUVIER, Arnaud, Le corps de l'artillerie en France de Louvois à la Révolution française : problèmes et méthodes, dir. J.-P. BoIs.

\section{Histoire contemporaine}

BEGUE, Sandrine, La fin de l'empire indoportugais (1933-1961): mythes et réalités de l'Estado da India, dir. J. WEBER.

Bossard, Sébastien, La Grèce face à l'opération des dardanelles d'août 1914 à octobre 1915, dir. J. WEBER.

DuFour, Isabelle, Les religieuses en mission ouvrière dans le diocèse de Nantes, dir. M. LAUNAY.

EvstafiEva, Viktoria, La question allemande dans les relations entre la France et l'URSS pendant la guerre froide " stalinienne »1945-1953, dir. F. Bozo.

GaROChE, Brieux, Taïwan, enjeu de la guerre froide 1949-1955, dir. J. WEBER.

SAUZEAU, Grégory, Le Dominion sud-africain, 1910-1924: naissance et reconnaissance d'une nation. Les difficultés de l'Union sud-africaine à entrer en guerre, 1914, dir. J. WEBER.

Stanescou, Thomas, Pacifisme et mouvement de paix en France 1975-1987, dir. F. Bozo.

\section{9}

\section{Histoire ancienne}

QUEBRIAC, Chrystelle, Les importations méditerranéennes en Gaule à l'âge du fer : le carrefour Rhône-Seine-Loire, dir. J.-R. JANNOT.

Corson, Sophie, Les bronzes antiques du Musée Dobrée Nantes, dir. J. PEYRAS.

DuBIN, Jérôme, L'oeuvre d'Arnobe dans la littérature apologétique latine, dir. J. PEYRAS.

\section{Histoire moderne}


Bodiguel, Hervé, Les problèmes posés à la présence française dans l'Empire Ottoman de 1768 à 1793, dir. C. HERMANN.

BONNET, Natacha, Étude de familles angevines et nantaises possessionnées à Saint-Domingue et en Métropole au

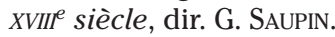

JoLIN, Nicolas, Les capitaines négriers à Nantes pendant la période 1717-1738, dir. G. SAUPIN.

PINEAU, Laure, L'élite négociante nantaise dans le second tiers du XVIII et au début du XIXe siècle : 1763-1815 : à partir de l'exemple de six familles liées par la mariage et les affaires: Drouin, Bouteiller, Chaurand, Deurbroucq, Berthrand, Lincoln, dir. G. SAUPIN.

GABARD, Véronique, Étude d'une petite ville de l'estuaire de la Loire au XVIII siècle et dans la première moitié du XIXe siècle, Paimbøuf de 1703 à 1856, dir. G. SAUPIN.

\section{Histoire contemporaine}

PEDRON, Frédéric, La politique extérieure du Bangladesh 1971-1975, dir. J. WEBER.

Collineau, Pascal, Les missionnaires catholiques et le mythe du Yunnan: actions, réactions et contributions des Pères des missions étrangères de Paris à une désillusion française (1843-1910), dir. J. WEBER.

LOYER, Vincent, La participation du Japon à la Première Guerre mondiale, dir. J. WEBER.

Martin, Sybille, La politique de l'Allemagne vis-à-vis de la Pologne depuis la chute du Mur de Berlin (1989-1995), dir. J. WEBER.

Mathe, Stéphane, La guerre et la Révolution française, dir. J.-C. MARTIN.

ROUSSEAu, Nelly, Être Africain et chrétien : pléonasme ou paradoxe? ou le défi de l'inculturation, dir. M. LAUNAY.

SoHIER, Benoît, La guerre de Sécession vue par les diplomates et les militaires français, dir. F. Bozo.

\section{8}

\section{Histoire médiévale}

CHOTARD, Stéphanie, Les forêts bourguignonnes à la fin du Moyen Âge, dir. M. LE MENE.

\section{Histoire moderne}

LE CORRE, Jérôme, La défense des côtes bretonnes et les principes tactiques de la guerre littorale de 1689 à 1789, dir. J.-P. BoIs.

PERREON, Stéphane, L'armée en Bretagne au XVIII siècle 1689-1789, dir. J.-P. BoIs.

PiCAud, Sandrine, La petite guerre en Europe, XVI'-XVII $-X V I I I^{e}$ siècles : sources et méthodes, dir. J.-P. Bors.

\section{Histoire contemporaine}

BOURROUET, Jean, L'intervention militaire américaine en Bosnie, dir. J. WEBER.

Bousseau, Céline, Un siècle de coolietrade chinois (1840-1940), dir. J. WEBER.

CARON, Pascal, Les interventions extérieures de la Bundeswehr depuis la réunification allemande, dir. J. WEBER.

COQuel, Muriel, Les missions étrangères de Paris en Inde 1776, 1836, 1886, les étapes du devoir accompli? 1785-1799: Négociations des obstacles, dir. J. WeBER. SEPRE, Sandrine, Le Pandit Nehru et le non-alignement 1947-1964, dir. J. WEBER.

\section{Mémoires de Maîtrise - Master 1}

\section{5}

\section{Histoire de l'art}

Audic, Sandrine, De l'archipel nantais à l'île de Nantes. L'urbanisation d'un territoire devenu stratégique, du milieu du XIXe siècle à nos jours, dir. A. BONNET.

GloAguen, Laetitia, Camille Bryen : un poète-peintre (1927-1977), dir. P.-L. RINUY.

GMAR, Katia, La spécificité de l'orfêvrerie nantaise au XVIII ${ }^{e}$ siècle, dir. H. RousTEAU-CHAMBON.

JANTON, Marie, Les difficultés des Jésuites de la mission du Madurai sous la pontificat de Benoît XV (1914-1922), dir. H. Rousteau-Chambon.

LASSALLE, Julien, Tristan Tzara dans son rapport à l'ouvre d'art : théorique, dir. P.-L. RinUY. 
Milliet, Morgane, Un livre d'heures à l'usage de Nantes le manuscrit 3072(R) de la médiathèque de Nantes, dir. J.M. Guillouet.

Plaisance, Morgane, Les expositions temporaires au Musée des Beaux Arts de Nantes et au Museu Contemporani de Barcelona (de 1995 à nos jours), dir. A. BONNET.

VACHER-SEYTOUX, Pierre, La réception en France des actionnistes viennois, dir. A. BONNET.

\section{Archéologie}

Guillotte, Céline, L'image des femmes, au Haut-Empire sur les monuments funéraires de la Gaule lyonnaise, dir. M. MONTEIL.

LUCAS, Nathalie, Les ex-voto à caractère médical en Gaule romaine : une forme d'art populaire, dir. M. MONTEIL.

\section{Histoire ancienne}

BonNIN, Franck, Les Sulpicii Galbae, entre République et Empire (167 av. J.-C.-68 apr. J.-C.), dir. T. PIEL.

BRIAND, Céline, Le corps des dieux en Grèce archaïque au travers des sources littéraires, dir. J. WILGAUX.

DALIBERT, Stéphane, Les possibilités de concertation entre les Augustes et les Césars dans la conduite et la direction des opérations militaires, dir. F. HURLET.

DuBors, Aurélie, Espace urbain et vie politique à Rome sous les Antonins et les Sévères à travers les sources littéraires, dir. T. PIEL.

Gallard, Delphine, Tarquin l'Ancien: étude d'un roi de Rome à travers la tradition littéraire, dir. T. PIEL.

LE CADRE, François, Le Royaume d'Axoum : recherche sur l'introduction du christianisme en Ethiopie au temps du roi Ezana, dir. T. PIEL.

Moreau, Mathieu, Entre idéologie et histoire : la cité étrusque dans les sources littéraires (VIII-III ${ }^{e}$ siècles av. J.-C.), dir. T. PIEL.

PIERREL, Élise, Les femmes de l'élite spartiate: mythes et réalités d'un parcours féminin au sein d'une de plus célèbres cités de la Grèce antique, dir. J. WILGAUX.

PuCEL, Marielle, Les groupes politiques à Athènes à l'époque classique, dir. J. WILGAUX.

\section{Histoire médiévale}

CROHEN, Arnaud, La mise à mort ritualisée chez les anciens Germains. vol. 1 : Meurtres exécutions et sacrifices humains, dir. P. JOSSERAND.

KuZ, Angelika, Le culte des saints dans l'ordre Teutonique, dir. P. JOSSERAND.

LESOT, Éliette, Bertrandon de la Broquière, espion bourguignon en terre d'islam : d'après le voyage d'Outremer (14321433), dir. J. TOLAN.

RABOT, Brice, Le prélèvement seigneurial en comté de Nantes à la fin du Moyen Âge (XIV -XV siècles), dir. J.-L. SARRAZIN.

Ronssin, Agnès, Un recueil d'exempla dans un manuscrit du Vatican : Édition de texte, dir. J. Tolan.

Simonneau, Romain, La croisade de Jean Germain, dir. J. Tolan.

\section{Histoire moderne}

BACON, Anne-Laure, Philippe Henri de Grimoard, un homme de l'ombre, dir. J.-P. BoIs.

Biennais, Céline, Économie et société rurale en Bas-Languedoc : étude du Compoix de Sigean, 1707, dir. D. LE PAGe.

Boutier, Jean-Claude, De la mer à la terre: le changement d'horizon des élites sociales de l'estuaire de la vie au XVII' siècle : étude d'histoire sociale à partir des archives notariales de SaintGilles et de Croix-de-Vie (1735-1792), dir. G. SAUPIN.

ColEno, François, Le baron de MesnilDurand ou la querelle des ordres (l'ordre mince ou l'ordre profond), dir. J.-P. Bors.

DAVID, Sylvain, La marine marchande française à travers les échanges commerciaux, entre le port de New York et les ports français dans la première moitié $d u$ XIX siècle, dir. G. SAUPIN.

DiAz-HERnANDEZ, Pedro Octavio, Miroirs troublés : opéra et politique en France à 
l'âge baroque 1682-1745, dir. Y. LIGNEREUX.

FRAPPIER, Roland, Mer et mort à travers cinq voyages au long cours (XVe-XVII ${ }^{e}$ siècles).

LaVILLE, Soizic, Les Noirs à Nantes de 1717-1740: aux origines d'une première vague d'immigration, dir. É. NoEL.

LE Roux, Léna, La colonie française de Cadix : 1773-1819, dir. G. SAUPIN.

MARCHAND, Guillaume, L'Helvétie au temps $d u$ Consulat, dir. J.-P. Bors.

NAULEAU, Anaïs, Les gens de la Sèvre nantaise au XVIII siècle, dir. G. SAUPIN.

Remaud, Julien, Jean Aulneau de la Maison Neuve (1677-1763), bourgeois rural de Bessay: La terre et les fondements de la puissance locale dans une petite paroisse du Bas-Poitou au début du

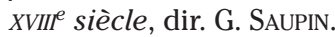

SAUCEY, Anne-Laure, Apprentis et apprentissage à Nantes à la fin $\mathrm{du}_{\mathrm{XVIII}}^{e}$ siècle, dir. D. Le PAGE.

\section{Histoire contemporaine}

Aumand, Julien, Le débat en France sur l'arme nucléaire française depuis le 11 septembre 2001, dir. F. Bozo.

AunEau, Olivier, La pénétration européenne au Maroc de la conférence de Madrid (1880) au protectorat (1912), dir. B. GioRGi-Mignot.

BARON, Amélie, La crise de la démocratie en Haïti sous le second mandat de Jean-Bernard Aristide (2000-2004), dir. L. JALABERT.

BENGHERADA, Ajib, La rétrocession de Hong Kong à la Chine vue par la presse française (11 décembre 1996-24 mai 1998), dir. J. WEBER.

BERGOUTS, Arnaud, Les diplomates français face à l'évolution du régime franquiste, 1966-1973, dir. M. CATALA.

Bouedo, Philippe, Le débat autour du second porte-avions français 2000-2005, dir. F. Bozo.

Bregeon, Cécile, La vision des femmes dans la presse nantaise, 1896-1906, dir. B. JOLY.

CARDINAL, Elsa, L'enseignement primaire et la question linguistique au Sénégal de 1945 à 1981, dir. A. TIREFORT.
Denizon, Pierre, La Transcaucasie, à travers les archives du Consulat de France à Tiflis : 1894-1918, dir. F. Bozo.

FouQuET, Julie, Les casinos en Vendée de 1876 à nos jours : leur impact sur le développement du département, dir. B. JolY.

GERART, Marie-Laure, Les conflits du travail à Pondichéry en 1936, dir. J. WEBER.

GUICHARD, Benoît, Le problème de la décolonisation dans la presse portugaise après l'opération indienne contre Goa, dir. J. WEBER.

Hamann, Laure, Les difficultés des Jésuites de la mission du Madurai sous la pontificat de Benoît XV (1914-1922), dir. J. WEBER.

HARDY, Daniel, Le diocèse de Nantes sous l'épiscopat de Monseigneur Michel Vial (1966-1982), dir. M. LAUNAY.

HERRIER, Anne, La question de Tanger, un aspect des relations franco-espagnoles de 1919 à 1923, dir. J. WEBER.

Hogrel, Soazic, La politique de gestion de la main-d'œuvre marocaines par le général Nogues 1936-1943, dir. M. CATALA.

JAGOT, Agnès, La diplomatie intérieure de la Yougoslavie de 1965 à 1974 vue par la diplomatie française, dir. F. Bozo.

JaUlin, Sylvain, Le Pakistan de Pervez Musharraf : du coup d'État militaire (12 octobre 1999) aux attentats du 11 septembre 2001, dir. J. WEBER.

LaIDIN, Fabien, La propagande française en direction de l'allié britannique entre 1914 et 1920, dir. M. CATALA.

LAMBERT, Sandrine, Le maintien de l'ordre à Nantes sous l'Occupation, dir. B. JoLY.

LiBouthet, Marion, Le Laos et le Laotien dans les récits de voyage, les revues et la littérature (1861-1914), dir. J. WEBER.

Methon, Cédric, La société soviétique sous Brejnev vue par la diplomatie française (1964-1974), dir. F. Bozo.

MonNoIR, Vincent, Images de l'Afrique dans l'œuvre de Georges Simenon, dir. L. JALABERT.

Moutoussamy, Mathilde, L'annexion du Sikkim à l'Union indienne, à travers la presse internationale 1973-1975, dir. J. WEBER. 
PerRaudeau, Amélie, L'industrie minière du Sud du Pays de Galles et l'organisation du patronat 1850-1898, dir. B. JOLY.

POUTEAU, Luc, Le débat régional en Loire Atlantique, du début des années 1970 au milieu des années 1980 à travers la presse locale, dir. C. POMEYRols.

VALETTE, Vassia Emmanuel, L'image d'Israël en France de septembre 2000 à novembre 2004. Relations franco-israéliennes et perceptions françaises d'Israël, dir. F. Bozo.

WROBLEWSKA, Malgorzata, L'élargissement de l'OTAN à l'Est à travers la presse française 1993-1999: le cas de la Pologne, La Croix, Le Canard Enchaîné, Le Figaro, Le Monde, Le Monde Diplomatique, Le Point, L'Humanité, Libération, dir. J. WEBER.

ZEo, Antoine, Français et musulmans dans l'Algérie coloniale à travers la littérature, 1899-1939, dir. J. WEBER.

\section{4}

\section{Histoire de l'art}

ARAujo, Frédéric, La représentation des fauves dans l'œuvre de Delacroix 18401863, dir. A. BonNET.

Avrilla, Maude, La Sphinge du Jardin des Tarots de Niki de Saint-Phalle, dir. A. BONNET.

BALEYDIER, Agnès, Images de la gladiature en Gaule romaine, dir. A. Bonnet.

DAOUDAL, Solène, Sirènes romanes en Poitou Xf-XIl siècles : avatars sculptés d'une figure mythique, dir. J.-M. GuILLOUET.

Guinebaud, Simon, L'orientalisation des armées séleucides : entre traditions macédoniennes et héritages achéménides, dir. I. Pimouguet-PEDARRos.

HAMON, Garance, Allan Sekula : la représentation des phénomènes économiques globaux dans la photographie contemporaine, dir. A. BONNET.

LE Coz, Vincent, Les armées Lagides à la Haute époque hellénistique, dir. I. Pimouguet-PedarRos.

LE Magadure, Évelyne, Autour de Jérôme Bosch : le "Saint Christophe » du musée
Dobrée de Nantes : un essai d'attribution, dir. J.-M. GuILLOUET.

PILARD, Jean-Jacques, Georges Brisson, peintre nantis (1902-1980) : la quête de la modernité en Province, dir. A. BoNNET.

PINEAU, Caroline, L'église Sainte-Anne de Saint-Nazaire, dir. A. BonNET.

RENAUT, Julien, Le rôle militaire des populations barbares d'Orient en marge $d u$ monde hellénistique et leurs implications dans l'histoire des grands royaumes, dir. I. Pimouguet-PEDARRos.

VISONNEAU, Thomas, Guerre et religion chez les Séleucides, dir. I. PImOUGueTPEDARROS.

\section{Archéologie}

DENNE, Stella, Les implantations galloromaines dans leur rapport avec l'environnement, dans le Nord Ouest du pays Picton, fer av. J.-C.-Ive apr. J.-C., dir. M. MONTEIL.

\section{Histoire ancienne}

Chauvire, Cécile, Recherches sur la "gens " Fabia 509-264 av. J.-C., dir. T. PIEL.

DuboIs, Céline, Les rites de naissance en Grèce aux époques classique et hellénistique, dir. J. WILGAUX.

Guillet, Ludivine, Le travestissement dans les mythes grecs, dir. J. WILGAUX.

LEVEQUE, Stephen, Les migrations Sabelliennes dans le Latium aux $v^{e}$ et IV siècles av. J.-C., dir. T. PIEL.

Peroys, Aurélien, Héphaitos à Athènes aux périodes archaïque et classique, dir. J. WiLgauX.

Peytral, Kevin, Le suicide en Grèce ancienne, dir. J. WILGAUX.

STASSINET-CAZENEUVE, Recherches sur Tarquin le Superbe, dir. T. PIEL.

TOUGERON, Anne-claire, Les mosaïques sassanides, dir. T. PIEL.

\section{Histoire médiévale}

CHARRIER, Daphné, Le sanctuaire de SaintMichel et les pèlerinages occidentaux au Monte Gargano des origines à la fin de la période normande (Ve-XII siècles), dir. P. JOSSERAND. 
ChAudet, Guillaume, Islam et iconographie au Sénégal, dir. J. Tolan.

Cussonneau, Virginie, La vision chrétienne du monde musulman à travers l'image de Mahomet dans l'iconographie européenne du XI ${ }^{e}$ au XV $v^{e}$ siècle, dir. J. TOLAN.

FoURNIER, Caroline, Les bains dans le monde urbain andalusi espaces, formes et fonctions ( $X^{e}-X V^{e}$ siècles $)$, dir. C. MAZZOLI-GUINTARD.

GAILLARD, Marine, Le Temporel de l'Abbaye cistercienne de Villeneuve au Moyen Âge 1201-1540, dir. J.-L. SARRAZIN.

GUILLOREL, Karen, Guérir et ressusciter au $X I I^{e}$ siècle par la main de Saint James : étude d'un recueil de miracles de l'abbaye de Reading, dir. P. JOSSERAND.

KoBUSINSKI, Juliette, La perception des faits économiques chez les chroniqueurs français de la fin du Moyen Âge, dir. J.-L. SARRAZIN.

LALY, Solène, Musulmans et “dhimmi/s »: espaces de sociabilité dans les villes d'al-Andalous d'après la littérature juridique IXe-XVe siècles, dir. C. MAzzoliGUINTARD.

RATURAT, Mélinée, Le Temporel de l'abbaye de Blanche Couronne au Moyen Âge, dir. J.-L. SARRAZIN.

\section{Histoire moderne}

BRAUD, David, La reconstruction de Cholet et de Chemillé : deux communes des Mauges après la guerre de Vendée (1795-1815), dir. J.-P. Bols.

BREHERET, Morgane, Nantes et son espace fluvial au XVIII siècle : étude de la société urbaine et de ses relations avec un espace physique original vital et très concurrentiel, dir. G. SAUPIN.

DucEPT, Alexandre, L'influence de Fontenay le Comte sur son " pays " au XVIII siècle (étude de la répartition sociale et de l'attraction de population dans la petite ville du Bas-Poitou de 1716 à 1792), dir. G. SAUPIN.

JAMIN, Lucie, Le Faubourg Sud de Nantes au XVIII siècle différents aspects d'un espace préurbain, dir. G. SAUPIN.
LE GALL, Vincent, Les officiers nantais aux lendemains de la Ligue, dir. D. LE PAGE.

LEVEQUE, Antoine, Entre orientalisme et interventionnisme : la mission du baron de Tott vue à travers le journal de bord du chirurgien Hollande, dir. J.-P. Bors.

Malllot, Véronique, Philippe de Brion, amiral de France sous le règne de François 1er (1492-1543), dir. D. LE PAGE.

PERROCHEAU, Richard, L'ambassade de Rome du cardinal de Bernis sous le pontificat de Clément XIV, 1769-1774, dir. J.-P. Bors.

RenouX, Michelle, La Chapelle/sur Erdre et Nantes au XVIII siècle ouverture ou isolement?, dir. D. LE PAGE.

SABLE, Mickaël, Une famille d'Irlandais en France au XVIII siècle : les Macnemara, dir. É. NOEL.

\section{Histoire contemporaine}

AUBERT, François, La politique étrangère de la Belgique entre atlantisme et construction européenne, avril 1954juillet 1958 vue par les diplomates français, dir. F. Bozo.

BARON, Solène, Les immigrants européens en Afrique du Sud pendant l'entre-deuxguerres, dir. A. TIREFORT.

BERNARD, Jérôme, Les événements en Côte d'lvoire de 1999 à 2003, vus par la presse africaine (Jeune Afrique l'Intelligent, Afrique-Asie, Le Soleil), dir. A. TIREFORT.

BolteAu, Teddy, La famine de 1998 dans le Sud Soudan : histoire d'un drame humain passé sous silence, dir. J. WEBER.

BotosoA, Fenozara Marie Manuela, La crise politique à Madagascar en 2002 : analyse à travers la presse française et africaine, dir. A. TIREFORT.

BOUCHERIE, Caroline, La police à Nantes sous le Second Empire, dir. B. Joly.

BOULVARD, Olivier, La figure de l'autre (Afrique, Asie) dans la chanson francophone contemporaine, de la fin des années 70 à nos jours, dir. A. TIREFORT.

BRIAND, Marine, La marche du sel et le second mouvement de désobéissance civile mené par Gandhi, d'après The Times, dir. J. WEBER. 
BROusSE, Laëtitia, Les étrangers de la région nantaise vus à travers "Le Phare de la Loire, de Bretagne et de Vendée », pendant l'entre-deux-guerres, dir. A. TIREFORT.

BuchART, Sylvanie, La Finlande dans les relations Est-Ouest, vue par les diplomates français de 1944 à 1956, dir. F. Bozo.

CARro, Stéphanie, Sport et politique : les Jeux Olympiques de Moscou (1980) vus par la République Démocratique Allemande, dir. F. Bozo.

CORBEL, Céline, La dictature en Argentine sous Videla au travers de la presse espagnole (1976-1981), dir. J. WEBER.

DAVID, Véronique, Les représentations $d u$ Sahara à travers l'illustration 18801920, dir. A. TIREFORT.

FERRE, Stéphanie, L'insurrection de Mahé en 1948, dir. J. WEBER.

GERARD, Jean-Jacques, Les milieux d'affaire nantais et le droit de visite (18301848), dir. J. WEBER.

GuICHARD, Aurélie, L'histoire de la colonisation et de la décolonisation dans les manuels d'histoire du collège des années 1970 à nos jours, dir. A. TIREFORT.

HERBET, Emmanuelle, Les répercussions de l'affaire de Goa sur la communauté internationale, année 1961, dir. J. WEBER.

JARRY, Érika, L'église catholique indienne face à la montée du fondamentalisme hindou : 1993-2003, dir. J. WEBER.

LAISNE, Pierre, La politique libanaise de la France de 1981 à 1985, dir. J. WEBER.

LASTENNET, Gaëlle, La seconde guerre de Tchétchénie vue par l'opinion publique française (1999-2003), dir. F. Bozo.

Le Marchand-Kamokwe, Cécile, L'indépendance du Cameroun : étude de la confrontation idéologique à travers la presse des colons et des colonisés (19551960), dir. A. TIREFORT.

LEAU, Cédric, Les élections générales indiennes de 1951-1952 vues par la diplomatie française, dir. J. WEBER.

LEBLAIS, Anaïs, La situation politique et administrative à Madagascar de 1956 à 1962, dir. A. TIREFORT.
LEDUC, Isabelle, Le naufrage du Saint-Philibert (14 juin 1931), dir. R. FABRE.

LEMOINE, Émilie, La vision française de l'élection présidentielle 2000 aux ÉtatsUnis, dir. F. Bozo.

LERAY, Céline, Les Marocains et l'école française de Casablanca entre 1920 et 1942, dir. J. WEBER.

LOMBARDINI, Audren, La criminalité en Loire-Inférieure (1871-1881) : aspects et facteurs, dir. R. FABRE.

MAIGA, Aminata, Les sociétés Ouest africaines du XIX siècle : le regard de quelques voyageurs européens, dir. A. TIREFORT.

Martin, Amélie, Étude de la population ambulante de Nantes et de son intégration durant l'entre-deux-guerres (nomades, forains, circassiens, marchands ambulants), dir. A. TIREFORT.

MILARET, Tonja, Action et politique culturelle de la France en Afrique du Sud de 1930 à 1945, dir. J. WEBER.

Petiot, Samuel, La politique culturelle française au Sénégal depuis l'indépendance : le centre culturel français de Dakar, dir. A. TIREFORT.

PIERS, Xavier, Le particularisme alaouite 1920-1930, dir. J. WEBER.

POIRIER, Thomas, La France vue par Scînteia sous de Gaulle : 1958-1969, dir. F. Bozo

RAINGEARD, Pierre, Les missionnaires Montfortains dans le diocèse de Luçon 18161856 d'après les chroniques de leurs missions (1816-1851), dir. R. FABRE.

REMAUD, Benoît, Le Parti Social Français en Loire-Inférieure (janvier 1937-mai 1940), dir. C. PomeYRols.

Robin, Fabien, La vision des diplomates français sur la politique extérieure yougoslave de 1953 à 1961, dir. F. Bozo.

Rousse, Cédric, L'action culturelle française en Italie entre 1958 et 1969 : établissements français, enseignement et diffusion culturelle, dir. J. WEBER.

SEDDIKI, Smaïn, La rivalité franco-britannique en Égypte 1876-11 juillet 1882 vue par le Times et le Temps, dir. J. WEBER. 
TAILLIER, Nicolas, Le Soudan et le terrorisme international de juin 1989 à fin 2001, dir. J. WEBER.

TAUVEL, Lucas, La France et la guerre du Kosovo, opinions et débats (1998-2000), dir. F. Bozo.

TERRIEn, Guillaume, Regards croisés sur la guerre d'Algérie (1945-1962). L'information orale dans l'Ouest-France et le témoignage oral : de l'engagement à la mémoire, dir. A. TIREFORT.

VIGNAULT, Thomas, Le thème de la piraterie au Tonkin dans la presse française et la littérature coloniale de 1872 à 1897, dir. J. WEBER.

VINET, Élise, La presse et la montée du nationalisme en Tunisie de 1919 à 1926, dir. J. WEBER.

\section{3}

\section{Histoire de l'art}

Brost, Pauline, Le Cours Cambronne. Entre utopie et réalité, dir. H. ROUSTEAuCHAMBon.

Dıт, Muriel, La représentation de la danse de Carpeaux à Matisse 18701910, dir. A. BonNET.

GEINDREAU, Anaelle, Jacques V Gabriel dans le Val de Loire, dir. H. RousteAuCHAMBON.

Hauguel, Amélie, L'école régionale des Beaux Arts de Nantes et la question de l'enseignement artistique nantais : étude historique 1904-1945, dir. A. BONNET.

Huchet, Valérie, Le théâtre et la peinture au XIXe siècle, dir. A. BonNET.

Nicolo, Olivia, La société des amis des Arts de Nantes (1890-1920), dir. A. BoNNET.

PACOR, Élodie, Les façades sculptées des maisons en pans de bois à la fin du Moyen Âge à Nantes, à travers les exemples de deux poteaux-corniers conservés au Musée Thomas Dobrée, dir. J.-M. GuILLOUET.

Pageau, Cédric, Pierrick Sorin : un artiste populaire, dir. A. BONNET.

REZGUI, Dounia, La représentation des prostituées dans la peinture du XIX siècle : réception, critique et évolution du thème 1847-1896, dir. A. BONNET.
Roux, Sébastien, Le manoir de Chassay dans l'architecture du comté nantais, au XVI ${ }^{e}$ siècle, dir. H. ROUSTEAU-CHAMBON.

\section{Archéologie}

CoRbion, Aline, Les bijoux à l'époque gallo-romaine dans l'Ouest de la France, dir. M. MONTEIL.

\section{Histoire ancienne}

BERTHOME, Sandrine, Les représentations d'Athéna dans la céramique attique des $v^{e}$ et $v^{e}$ siècles, dir. C. GRANDJEAN.

CHARLES, Alexandre, Alexandre et la religion, dir. C. GRANDJEAN.

FREOUR, Marie, La naissance de l'alphabet grec : histoire d'un débat du début des années 1950 au début des années 2000, dir. C. GRANDJEAN.

HuBERT, Aurélie, Les Asclépieia à Epidaure aux IV et III siècles avant J.-C., dir. C. GRANDJEAN.

LANDAIS, Gaëlle, Les amazones dans l'art gréco-romain : première approche, dir. T. PIEL.

LE BAUT, Florence, Épargnes et banques à Athènes de la fin du ve siècle avant J.-C. à la fin du IV siècle avant J.-C., dir. C. GRANDJEAN.

LE Foulgoc, Nelly, Femme et guerre en Grèce ancienne de l'époque archaïque à l'époque classique, dir. C. GRANDJEAN.

\section{Histoire médiévale}

AnToIne, Alexandre, Jérusalem et la société catalane aux $X^{e}$ et $X I^{e}$ siècles, dir. P. JosSERAND.

BuIN, Anthony, Les chrétiens d'Orient au regard de Guillaume de Tyr, dir. P. JosSERAND.

Bossis, Carole, Les entrées royales sous les règnes de Jean II et Henri IV de Castille, selon les chroniques de l'époque, dir. P. JOSSERAND.

GIRARD, Julien, Le temporel du Prieuré de la Chaize-le-Viconte (1069-1556), dir. J.-L. SARRAZIN.

Guiot, Laurence, L'image des mosquées dans les sources chrétiennes du XIII siècle, dir. J. TOLAN. 
GuIsoliA, Yolaine, La notion de pèlerinage au XII siècle à travers les écrits de saint Bernard de Clairvaux, dir. P. JOSSERAND.

JAUNET, Julien, La vie de frontière entre la Castille et le royaume de Grenade à travers les Miracles de Pedro Marin (fin XIII siècle), dir. P. JOSSERAND.

TARDIVEAU, Bertrand, Un regard sur les limbes occidentales de la Chrétienté latine : Giraud de Barri (1146-1223), dir. P. JOSSERAND.

\section{Histoire moderne}

BELKIR, Malik, La représentation des gens d'outre-mer dans les élites nantaises au XVII' siècle (1716-1791), dir. É. NoEL.

BONNET, Jérôme, La famille Richard au $\mathrm{XVIII}^{e}$ siècle : l'exceptionnelle réussite économique et sociale par le grand commerce maritime, dir. G. SAUPIN.

BOUTERA, David, Les bohémiens en Bretagne du XVI ${ }^{e}$ au XVIII ${ }^{e}$ siècle, dir. D. LE PAGE.

BROCHARD, Cécile, Violence et mentalités dans la société bretonne d'après les lettres de rémission (1516-1518), dir. D. LE PAGE.

Deschamps, Jacques, Le général Derrecagaix (1833-1915) et l'écriture militaire en France au XIX $X^{e}$ siècle : la Guerre moderne 1885.

Dousset, Sandrine, Un jeu urbain, le Papegault dans les villes bretonnes de la fin du XV $V^{e}$ siècle à la fin $d u X V I I I^{e}$ siècle, dir. D. LE PAGE.

FLODERER, Juliette, Ancenis au XVIII siècle : étude sociale d'une petite ville et de sa capacité d'attraction (1737-1791), dir. G. SAUPIN.

Guilbaud, Christelle, La famille de Sesmaisons en Normandie aux XVII et XVIII siècles, dir. D. LE PAGE.

GUIMAS, Romain, La perception de l'armée à Nantes sous la Restauration, dir. J.-P. Bols.

HERAULT, Cécile, Heurs et malheurs d'une famille négociante nantaise d'origine basque : les Darquistade aux XVII et XVIII $e^{e}$ siècles, dir. G. SAUPIN.

HERVE, Étienne, Une famille de négociantsarmateurs irlandais à Nantes au XVIII siècle : les O’Shiell (1689-1799), dir. É. NOEL.

KNUCHEL, Geoffroy, La correspondance d'Aristide Henry Crouzet, officier supérieur sous la Troisième République (1870-1887), dir. J.-P. Bols.

Kunzelmann, Philippe, La guerre du Paraguay dans les sources diplomatiques françaises (1864-1870), dir. C. HERMANN.

Le DOARE, Catherine, Les obstacles à la politique d'embellissement du maire Gérard Mellier (1720-1729) à Nantes et leurs conséquences, dir. G. SAUPIN.

LE Goff, Ronan, L'intégration des personnes de couleur à Nantes au XVIII siècle, dir. É. NOEL.

Legagneux, Anne-Laure, Criminalité et société d'après les dossiers criminels de la sénéchaussée de Guérande (17151750), dir. G. SAUPIN.

LoussouARn, Virginie, Le Parlement de Bretagne 1554-1600 : carrières des présidents et des conseillers, dir. D. LE PAGE.

MACAUD, Marie-Laure, Foires et marchés à la Roche-sur-Yon 1804-1904, dir. G. SAUPIN.

MALEGOL, Raphaël, La reconstruction $d u$ quartier nantais de la Casserie suite à l'incendie, dir. G. SAUPIN.

PAITIER, Émilie, L'image de l'armée pendant la guerre de 1870 à travers La Gazette de l'Ouest, dir. J.-P. Bors.

PInEAu, Cécile, Les États de Bretagne dans la seconde moitié du XVI siècle, dir. D. LE PAGE.

POIRIER, Amandine, Mably et la nature du pouvoir monarchique : recherche d'un modèle légitime dans la lutte contre le pouvoir despotique, dir. M. BELISSA.

SouffLET, Yannick, Les négociants nantais et l'architecture : le quai de la Fosse (1735-1755), dir. G. SAUPIN.

YoкоYама, Nao, Le noyau du conseil des bourgeois de Nantes au milieu du XVI siècle, dir. G. SAUPIN.

\section{Histoire contemporaine}


AubINEAU, Nathanaëlle, Représentations de Madagascar dans les Expositions coloniales 1885-1931, dir. A. TIREFORT.

AumONNIER, Amaury, L'intégration de la Turquie dans l'Union Européenne et l'opinion française du sommet d'Helsinki 1999 au sommet de Copenhague 2002, dir. F. Bozo.

Beduneau, Samuel, Le débat en France sur les rapports franco-allemands et les problèmes de l'Union Européenne (de 1995 à 2003), dir. F. Bozo.

BoISDRON, Matthieu, La perception de la crise des années Trente en Roumanie par les diplomates français en poste à Bucarest, dir. B. JolY.

Bomme, Thomas, Le Monde, Le Figaro et l'Humanité face au Printemps de Prague et à sa répression (janvier-août 1968), dir. J. WEBER.

BOUINEAU, Alexandre, Histoire d'une élite sénégalaise. Lycée Lamine Gueye, 19031960, dir. A. TIREFORT.

BOUTTIER-SIMON, Marie-Josée, L'Église SainteThérèse de Nantes, dir. M. LaunaY.

BRETON, Annelise, La diplomatie italienne et la crise de Suez : les rapports francoitaliens, dir. F. Bozo.

CARER, Solenn, L'Inde britannique à son apogée vue par les écrivains voyageurs français (1858-1914), dir. J. WEBER.

Снавот, Benoît, Le problème indien à Madagascar de 1945 à 1956 (Tentative d'analyse du discours anti-indien à travers la presse malgache et francophone), dir. (A.) TIREFORT.

ChAMPENoIs, Grégoire, La politique française au Maghreb (Maroc, Algérie, Tunisie) entre 1953 et 1958 à travers la presse espagnole, dir. J. WEBER.

CHAPON, Amanda, Une tribu de la Seguia el Hamra au début du protectorat français au Maroc 1912-1919 à partir des archives françaises, dir. J. WEBER.

David, Nicolas, La société soviétique khrouchtchérienne (1953-1963) vue par les diplomates français, dir. F. Bozo.

Desmars-Chausse, Marie-France, Un siècle d'histoire de la congrégation des soeurs de l'instruction chrétienne de Saint-Gildas-des-Bois 1807-1914, dir. M. LaunaY.
DorTtEAU, Nolwenn, La gestion des conflits sociaux ou une histoire commune de Kuhlmann et Paimbouf (1919-1997), dir. B. JolY.

Dore, Régis, L'enseignement de l'Histoire au Sénégal : le Primaire et le Collège dans la deuxième moitié du XXe siècle, dir. A. TIREFORT.

FRANGEUL, Frédéric, La politique de Lord Mountbatten en Inde vue par la presse britannique (février-août 1947), dir. J. WEBER.

GaILliot, Pauline, La conférence de Bandoung vue par la presse française, dir. J. WEBER.

GANUCHAUD, Laure, Les Khmers rouges de Pol Pot : résistances et oppositions du 17 avril 1975 au 7 janvier 2002, dir. J. WEBER.

Gaudin, Julie, Des prêtes et des séminaristes Nantais rappelés en Algérie en 1956 témoignent, dir. M. LaUnAY.

HEURTIN, Linda, Histoire d'une congrégation religieuse nantaise au XIX $X^{e}$ siècle : les sœurs de l'Immaculée Conception de La Haye Mahéas, dir. M. LaUnAY.

HUBERT, Solène, Le putsch des généraux vu par la presse française, dir. J. WEBER.

Kunzelmann, Philippe, La guerre du Paraguay dans les sources diplomatiques françaises (1864-1870).

LE TEUFF, Florian, Les socialistes nantais et les gauches 1933-1937, dir. C. POMEYROLS.

LEBOURG, Pierre, Les débits de boisson à Nantes et dans la Loire-Inférieure au XIXe siècle (1800-1914), dir. B. JolY.

LEBRUN, Vanessa, L'opinion publique nantaise sous le gouvernement Daladier, avril 1938-mars 1940, dir. B. Joly.

LEMERLE, Arnaud, L'enseignement catholique dans le diocèse de Nantes, 19551971 : mutations institutionnelles et culturelles, dir. M. LAUNAY.

LESIEUR, Julien, Les ouvriers nantais de 1860 à 1870, dir. R. FABRE.

LoBrot, Caroline, La France, les Pays Bas et les problèmes de la construction européenne de 1958 à 1966, dir. F. Bozo.

LOQUIN, Frédéric, La bataille de Kargil (février 1999-octobre 1999), dir. J. WEBER. 
MACAUD, Marie-Laure, Foires et marchés à la Roche-sur-Yon 1804-1904.

PACTEAu-Bouchet, Marie-Laure, Saint-Laurent-sur-Sèvre " la ville sainte de la Vendée ». Histoire de la vie paroissiale de 1877 à 1915, dir. M. LAUNAY.

PEDRon, Nathalie, "Mars et les Arts", 1799-1873. Histoire d'une loge maçonnique nantaise, dir. C. Pomeyrols.

Pelletier, Jonathan, De l'Association Catholique de la Jeunesse Française aux mouvements spécialisés en Vendée 1920-1950, dir. M. LaunaY.

PElloquin, Anna, La présence française au Cambodge vue à travers le journal Le Temps de 1863 à 1897, dir. J. WEBER.

PINEL, Anthony, Nantes, l'abolition de l'esclavage et ses conséquences à la Réunion : 1848-1852, dir. J. WEBER.

PRIN, Sabine, La politique culturelle de la France en Afrique Noire et à Madagascar, au-delà des Indépendances, par le biais des centres culturels français, dir. A. TIREFORT.

QuEDILLAC, Laure, L'évolution de la notion de l'inceste en Angleterre et au Pays de Galles au XIXe siècle, dir. B. Joly.

RAYANT, Kevin, L'action culturelle française aux Pays Bas 1958-1969, dir. J. WEBER.

Roland, Nicolas de, Le débat en France sur l'élargissement de l'Union Européenne (1989-2002), dir. F. Bozo.

SAUGER, Mélinda, La diplomatie italienne et la Guerre d'Algérie de 1954 à 1957, dir. F. Bozo.

SINEL, Marie-Laure, L'Afrique Occidentale Française, à travers le journal L'Illustration de 1890 à 1931, dir. A. TIREFORT.

THOMAS, Pierre, La diplomatie française face à la seconde crise de Berlin 19581963 : à travers le fonds de Bonn, dir. F. Bozo.

Touchon, Nicolas, La vision des diplomates français sur l'évolution politique de la Yougoslavie de 1945 à 1953, dir. F. Bozo.

VALY, Fabienne, La place de l'Iran dans le nouveau grand jeu afghan, dir. J. WEBER.

\section{Histoire de l'art}

AUBERT, Émeline, Joseph Kosuth " Art comme idée comme idée », dir. A. BoNNET.

AUdUREAU, Sophie, Louis Roger (18741953) : un peintre officiel méconnu, dir. A. BONNET.

BARBARIT, Matthieu, Fortifications maritimes et aménagements portuaires en Asie mineure occidentale et méridionale aux époques classique et hellénistique, dir. I. Pimouguet-PEDARRos.

DANIEAU, Sophie, Les pratiques guerrières chez les peuples non grecs, à travers les sources grecques (littéraires et iconographiques). Des guerres médiques à l'année 338, dir. I. Pimouguet-PEDARRos.

Delauré, Claire, L'architecture archéménide et son influence aux époques classique et hellénistique en Asie mineure occidentale, dir. I. PIMOUGUET-PEDARROS.

DOUCET, Maude, Les non-combattants et la guerre dans le monde grec des cités à l'époque classique (490-338 av. J.-C.), dir. I. Pimouguet-PEDARRos.

Haumon, Stéphanie, Questions sur l'art naïf à travers les itinéraires de deux peintres régionaux: Simone Le Moigne et Maurice Loirand, dir. A. BONNET.

JAGOT, Hélène, L'image des gaulois dans l'art au XIXe siècle : problèmes iconographiques et utilisations idéologiques, dir. A. Bonnet.

Juvin, David, La guerre dans les discours de Démosthène, dir. I. Pimouguet-PedARROS.

Le DARZ, Émilie, Peinture et mode de la Révolution à l'Empire : le phénomène des Incroyables et des Merveilles, dir. A. BONNET.

Quernez, Olivier, Le Fonds régional d'art contemporain des Pays de la Loire : collection et politique d'acquisition (1983-2001), dir. A. BonNET.

Rivalland, Anne-Sophie, La cité d'Halicarnasse à l'époque classique : une topographie du pouvoir, dir. I. PIMOUGUET-PEDARROS.

\section{Histoire ancienne}


ELHIAR, Isabelle, L'intégration du culte d'Isis à Rome, vue à travers les auteurs latins, grecs et chrétiens, du frr siècle avant J.C. au ve siècle après J.-C., dir. É. GUERBER.

GADE, Perrine, Le bagage des armées grecques, dir. C. GRANDJEAN.

GAUTIER, Fabien, Sentiments anti-romains et philo-romains en Asie Mineure à la fin de la République : l'exemple des guerres d'Aristonicos, de Mithridate et de Labienus, dir. É. GUERBER.

GIRAUDEAU, Estelle, Les relations diplomatiques entre Argos et Athènes à l'époque classique (490-336), dir. C. GRANDJEAN.

JuliEnNE, Adeline, Recherches sur Ixion, dir. C. GRANDJEAN.

MALARD, Maryannick, Le rôle politique et social des reines attalides, antigonides, séleucides et lagides à l'époque hellénistique, dir. C. GRANDJEAN.

PERIN, Fabrice, La perception qu'avaient les Athéniens des Thebains à l'époque classique dans la littérature et le théâtre, dir. C. GRANDJEAN.

VENTROUX, Olivier, Les institutions politiques de Smyrne et Pergame d'après l'étude des inscriptions d'Auguste à Diocletien, dir. É. GUERBER.

\section{Histoire médiévale}

Furon, Christophe, Pouvoir et défense du territoire en Morée franque (12051430), dir. P. JOSSERAND.

GAuthey, Thomas, Le Bestiaire d'Aberdeen : un exemple de la culture savante médiévale entre spiritualité et politique, dir. J. TOLAN.

Gouguet, Guillaume, Armes d'hast et de choc à l'époque de Jean Froissart, dir. P. JOSSERAND.

Grosse, Marie, Le culte de Saint Brieuc en Bretagne au Moyen Âge : du IX $\mathrm{X}^{e}$ uu XIII siècle, dir. J.-L. SARRAZIN.

Guibot, Emmanuelle, La châtellenie de Champagné-les-Marais (1442-1561), dir. J.-L. SARRAZIN.

LAGET, Frédérique, "De Paour et crainte d'estre tuez ": la peur en Bretagne à la fin du Moyen Âge (vers 1340-vers 1515), dir. J.-L. SARRAZIN.
LE NoAC'H, Charlotte, Le Temporel de la commanderie de Coudrie du XII au XV siècle, dir. J.-L. SARRAzIN.

LEBRETON, Gwenn, Les ordres militaires ibériques et les musulmans aux XIt -XIV siècles, dir. P. JOSSERAND.

Mussat, Aude, La sorcellerie dans le procès de Gilles de Rais, dir. J.-L. SARRAZIN.

Patouillere, François-René, La seigneurie d'Anetz (1390-1499), dir. J.-L. SARRAZIN.

SAILLARD, Anne-Laure, Culture et pouvoir royal en Castille sous les règnes d'Alphonse VIII et de Ferdinand III, dir. P. JosSERAND.

\section{Histoire moderne}

Ausıas, Huguette, Jean-Marie Déguignet (1834-1905) : l'itinéraire intellectuel d'un paysan bas-breton (d'après "L'Histoire de ma vie » de Jean-Marie Déguignet), dir. D. LE PAGE.

BARRE, Philippe, Les seigneuries de Lesnerac, Escoublac, Bois-Jollan et Tregrain au temps des guerres de religion (1543-1612), dir. D. LE PAGE.

BEGAUD, Stéphane, La représentation diplomatique française aux États-Unis et le conflit anglo-américain (1807-1815), dir. M. BELISSA.

BIZET, Christophe, La guerre voulue, la paix subie? L'ordre européen sous le Consulat, 1799-1804: d'après le "Moniteur Universel ", dir. M. BELISSA.

Bozec, Marion, Penser la guerre et la paix dans l'Encyclopédie de Diderot, dir. (M.) BELISSA.

Brenon, Céline, L'ascension sociale d'une famille de la noblesse bretonne (XVI XVIII siècle) : Les Sesmaisons (de), dir. D. LE PAGE.

CHEVRIER, Anne-Claire, La navigation et le commerce français au Portugal 18151854, dir. C. HERMANN.

DESCAMPS, Fabrice, Les relations francorusses au cours de la première guerre balkanique, d'après les archives $d u$ fonds Petrograd, dir. C. HERMAnN.

Guillard, Audrey, Tallemant des Réaux et les femmes du premier XVII siècle dans les Historiettes, dir. D. LE PAGE. 
HAREL, Nicolas, Du général de la trêve du Bas-Paimbouf (1730-1761) au général de paroisse de Paimbouf (1761-1789), dir. G. SAUPIN.

Jousseaume, Dominique, Les fonctions militaires de la ville de Nantes, 15981629, dir. G. SAUPIN.

LEBRAT, Soizic, L'Académie de musique et le Concert de Nantes au XVIII siècle, dir. G. SAUPIN.

PAIREAU, Valérie, Le traité de Saint-Germain 29 mars 1632 : Canada, France et Angleterre au début du XVII siècle, dir. J.-P. Bois.

PICHON, Olivia, Les hôtels aristocratiques à Nantes XVII ${ }^{e}$-XVIII ${ }^{e}$ siècles, dir. G. SAUPIN.

Rigaud, Héléna, Les activités et le personnel de la Chambre des Comptes de Bretagne sous la régence de Marie de Médicis 1610-1617, dir. D. Le PAGE.

Routhiau, Sandrine, La vie privée des officiers supérieurs de la Chambre des Comptes de Bretagne au début du règne de Louis XV 1715-1748, dir. D. Le PaGe.

SOLEILHET, Isabelle, Étude morphologique et sociale de la paroisse Saint Saturnin au XVII et au début du XVIII siècle, dir. G. SAUPIN.

VISSER, Joseph, Le Consulat de France à Baltimore sous l'exercice de Charles François Adrien Le Paulmier, chevalier d'Annemours, 1778-1793, dir. M. BELISSA.

Watremez, Florian, Mably et Rousseau au chevet de la Pologne, dir. M. BELISSA.

\section{Histoire contemporaine}

BARANGER, Aurélie, La France de Lionel Jospin vue par la Grande-Bretagne de Tony Blair, de 1997 à 2002, au travers de la presse, dir. F. Bozo.

BATARD, Amélie, La politique mise en ouvre par le Protectorat français au Maroc à l'égard du mouvement sioniste entre 1917 et 1925, dir. A. TIREFORT.

BAUDRY, David, Les relations franco-allemandes de 1905 à 1914 vues par deux journaux républicains nantais : Le Phare de La Loire et Le Populaire de Nantes, dir. C. POMEYROLS.
CaIGnON, Édith, L'Inde d'Indira Gandhi en état d'urgence, 1975-1977, à travers Le Times, dir. J. WEBER.

Cancouet, David, La politique indigène du résident Massicault en Tunisie (18861892), dir. J. WEBER.

CARRE, Bleuenn, The Anglo-Oriental Society for the Suppression of the Opium Trade, entre Grande-Bretagne et Chine, de 1874 à 1887, dir. J. WEBER.

CHAPAYs, Éric, Quand naissait la Baule, la paroisse d'Escoublac de 1870 à 1925, dir. M. LAUnAY.

GAUTIER, Céline, Les hospices civils de Nantes de la Révolution et de l'Empire: évolution du patrimoine immobilier 1790-1810, dir. M. LAUNAY.

GHostine, Marc, Confessionnalisme et vie politique au Liban sous la présidence de Charles Debbas (1926-1934), dir. J. WEBER.

Hamelin, Philippe, Domination coloniale et politique indigène en Algérie (18711891), dir. J. WEBER.

JARRY, Benjamin, La crise arménienne de 1895-96 dans l'Empire ottoman selon les diplomates français, dir. R. FABRE.

LAURIER, Mathieu, Gaston Veil (1868-1947) dans la vie politique et sociale nantaise : itinéraire d'un intellectuel affairé, dir. C. POMEYRols.

Le Fur, Yvon, Les accords de la Baie d'Along de juin 1948, dir. J. WeBER.

Le MoAn, Catherine, Les chemins de fer pendant la Seconde Guerre mondiale. Un exemple : l'Établissement SNCF de Belfort.

MARDEGAN, Sabine, Les Européens en Haute Volta de 1945 à 1960 : étude d'une société coloniale, dir. A. TIREFORT.

Pele, Gaëlle, La concession française de Tientsin : empiètements occidentaux et nationalisme chinois 1900-1917, dir. J. Weber.

Penlae, Marie, Le référendum de septembre 1958 en Algérie. Une population appelée à s'exprimer dans la guerre, dir. J. WEBER.

Penot, Pierre-Étienne, La Pologne et la loi martiale de 1981 à travers la presse

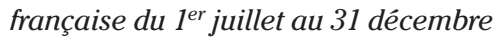
1981, dir. J. WEBER. 
Platov, Christine, La politique de Nikita Khrouchtchev pendant ses dernières années au pouvoir vu par le journal Le Monde 1961-1964, dir. R. FABRE.

Polisse, Hélène, La montée du nationalisme africain au Kenya (1944-1963), dir. A. TIREFORT.

Rault, Mathilde, La révolte des Cipayes vue par Le Times, dir. J. WEBER.

RauLT, Émilie, L'Indépendance de l'Inde et du Pakistan vue par la presse française (20 février 1947-30 janvier 1948), dir. J. WEBER.

REMAUD, Jeoffrey-Gaylord, L'Éthiopie de 1935 face à l'argumentaire colonial italien: mythe ou réalité d'un pays africain moderne, dir. A. TIREFORT.

Renou, Pierre, Le Djebel Druze 1914-1927, dir. J. WEBER.

RoBIN, Jean-Baptiste, Les relations entre les syndicats agricoles de Loire-Inférieure et le front paysan de 1934 à 1939, dir. C. POMEYROLS.

RoBineau, Anne, L'infiltration économique japonaise dans la colonie portugaise de Timor de 1936 à 1942, dir. J. WEBER.

RoEST, Angela, Représentations du corps féminin en France entre 1919 et 1925 : l'exemple des revues féminines et de la médecine usuelle, dir. A. TIREFORT.

SAICHE, Kamel, La justice et la police en Afrique Occidentale Française, dir. A. TIREFORT.

VERRON, Aurélie, Les mutations sociales au Sénégal dans la seconde moitié du XXe siècle à travers les romans de Sembene Ousmane, dir. A. TIREFORT.

\section{1}

\section{Histoire de l'art}

ChARLES, Damien, Les guerres d'anéantissement chez les Macédoniens durant les règnes de Philippe II et d'Alexandre Le Grand, dir. I. PIMOUGuet-PEDARRos.

DESHAYES, Nicolas, Le commandant militaire dans l'Anabase de Xénophon: expédition lointaine et émergence $d u$ chef de guerre, dir. I. Pimouguet-PedARROS.

\section{Histoire ancienne}

BEnNADJI, Samira, Paganisme et païens dans l'œuvre de Lactance, dir. J. PEYRAS.

Breton, Karine, La défense des Gaules au Iv siècle, dir. J. PEYRAS.

COUTAND, Nicolas, La magie dans l'Occident romain: les tablettes d'exécration, dir. J. PEYRAS.

GefFroy, Amélie, Aelius Aristide et Asclépios: Histoire religieuse et idéologique, dir. É. GUERBER.

LAUTRIDOU, Gaëlle, Les institutions politiques d'Éphèse sous l'Empire romain d'après les sources épigraphiques, dir. É. GUERBER.

\section{Histoire médiévale}

AubIN, Corinne, La figure du roi dans le manuscrit des Cantigas de Santa Maria d'Alphonse X Le Sage (1252-1284), dir. M. Clouzot.

BREJON, Fabrice, Abélard et l'Église (les relations d'un "intellectuel » avec l'autorité au XII siècle), dir. M. CLOUZOT.

CABRERA, Thomas, Saint Thomas d'Aquin et la musique du monde ou la musique et l'harmonie selon Saint Thomas d'Aquin (1225-1274), dir. M. Clouzot. ConTEncin, Anne-Soizic de, Le culte de Saint Mars au Moyen Âge dans l'Ouest de la France, dir. G. LouISE.

DeHIER, Elsa-Frédérique, Le patrimoine de l'Abbaye de Shrewsbury, $\mathrm{XI}^{e}-\mathrm{XIII} \mathrm{I}^{e}$ siècles. Une étude de son cartulaire, dir. G. LouISE.

LELONG, Mélanie, Une famille de la moyenne noblesse poitevine à la fin du Moyen Âge : les Appelvoisin, dir. J.L. SARRAZIN.

\section{Histoire moderne}

BARIBAUD, Laurence, Le diocèse nantais au temps de la Contre-Réforme catholique au XVII siècle, dir. D. LE PAGE.

BitEAu, Jérôme, Le Général Baron LouisArmand de Lespinay (1789-1869), dir. J.-P. Bois.

CARDOU, Nicolas, Un outil de distinction sociale : les mascarons de lîle Feydeau, dir. G. SAUPIN. 
DucRocQ, Audrey, Les aléas d'une famille de la noblesse nantaise au XVII et au XVIII siècle : la famille Boussineau, dir. G. SAUPIN.

FLEURANT, Virginie, Les gens de couleur à Nantes au XVIII siècle, dir. É. NoEL.

FREITAS, Céline de, La première rencontre de la France et des Républiques du Centre Amérique 1832-1852, dir. C. HERMANN.

GigaUd, Élisabeth, L' École de médecine de Nantes de 1808 à 1875.

JANTON, Laurence, Transcription des Archives du Boisarmant, dir. M. NASSIET.

JuHEL, Nolwenn, La pastorale d'un prêtre breton dans la seconde moitié du XVIII siècle : les sermons de René Cavaro de Kergorre, premier recteur du Croisic, dir. G. SAUPIN.

Legot-ThiRIEAU, Sylvie, Portrait d'un gentilhomme breton dans la seconde moitié du XVIII siècle : Jean-Baptiste Ménardeau II du nom, seigneur du Perray.

MAHE, Virginie, Costume et représentation sociale dans la Sénéchaussée de Guérande au XVIII e siècle (17251730/1785-1789).

MESNIER, Paul, La participation des troupes françaises à la campagne de Hongrie de 1664 contre les Ottomans. La bataille de Saint Gotthard (1er août 1664), dir. J.-P. BoIs.

PEDRON, Agnès, Les travaux publics dans une petite ville : le Croisic au XVIII siècle.

Rocheteau, Aurélien, Le Consulat de Boston de 1781 à 1793, dir. M. BELISSA.

Roussel, Lina, Les relations franco-espagnoles pendant la guerre de Sept Ans (1755-1763), dir. C. HERMANN.

\section{Histoire contemporaine}

AYMARD, Olivier, Tahiti au temps de l'affaire Pritchard vu par le Moniteur Universel et le Times (1842-1847), dir. J. WEBER.

BACHELIER, Delphine, La vision française de la Californie des années soixante, dir. F. Bozo.

BlanchaRD, Cyrille, En captivité. Une publication de Résistance démocrate-chrétienne dans la ville de Nantes (novembre 1940juillet 1941), dir. C. POMEYRoLS.

Boucault, Pascal, Les anciens combattants à Nantes durant l'entre-deuxguerres 1919-1939.

BRES, Arnaud, Les Sénégalais en Côted'Ivoire de 1893 à 1939, dir. A. TIREFORT.

Chaillou, Virginie, Des côtes de l'Inde aux plantations réunionnaises: les " coolies " en transition 1860-1882, dir. J. WEBER.

Chalmeau, Quitterie, Les Etudes et l'Europe 1947-1978, dir. M. LAUNAY.

CORNIC, Yann, Fraternité Matin et la politique étrangère de la Côte d'Ivoire 19651971, dir. A. TIREFORT.

Cosse, Thomas, La politique de l'information et la presse en Côte d'Ivoire de 1956 à 1973 : l'exemple de Fraternité Matin, dir. A. TIREFORT.

El Kenz, Myriam, Une lecture de l'Algérie au fil des expositions coloniales (1851-1931), dir. A. TIREFORT.

EsCHRICH, Nicolas, La rivalité franco-allemande au Chili, 1914-1939, dir. C. HERMANN.

EvaIN, Katell, Le pastorat du père Bigaré au Croisic (1840-1879), dir. M. LAUNAY.

Gergaud, Mathieu, La formation d'une élite malgache au collège Saint Michel de 1888 à nos jours, dir. A. TIREFORT.

GILBERT, Cédric, Blancs et noirs en Afrique noire française à travers la littérature coloniale de l'entre-deux-guerres, dir. J. WEBER.

GuicheTEAU, Amandine, Le mouvement autonomiste en Haute Djézireh (19361939), dir. J. WEBER.

GuIot, Hélène, L'image de l'Indien au travers des Annales de la Propagation de la Foi (1822-1857), dir. J. WEBER.

HARDY, Juliette, Les Français de Lengerich. Kommando extérieur du camp de concentration de Neuengamme, dir. J. WEBER.

Heraud, Béatrice, Le Pays de Galles, une réponse patriotique au déclenchement de la Première Guerre mondiale, dir. J.-C. MARTIN.

JUTEAU, Fanch, L'expansion coloniale européenne au XIX siècle dans la bande 
dessinée francophone publiée depuis 1980, dir. A. TIREFORT.

KANANE, Nora, Une nouvelle capitale pour le Penjab : Chandigarh 1947-1965, dir. J. WEBER.

KERIGNARD, Anthony, La vision française de l'affaire Lewinsky. Images de la société et des institutions américaines en France, dir. F. Bozo.

KERLOC'H, Alexandre, L'entrée de la RFA dans l'OTAN vue par la presse française - septembre 1954-mai 1955, dir. F. Bozo.

LAMOURET, Cyril, La rupture franco-égyptienne. De la crise de Suez au rétablissement des relations diplomatiques 1956-1963, dir. F. Bozo.

LELONG, Nathalie, Fernand Roy : une carrière diplomatique en Chine 1911-1946, dir. J. WEBER.

MERCIER, Cécile, Les pieds-noirs et l'exode de 1962 à travers la presse française, dir. J. WEBER.

Peignon, Florence, Septennat ou quinquennat : un débat politique et constitutionnel sous la Cinquième République (1974-1988), dir. J. WEBER.

PIFFETEAU, Sébastien, La Roumanie et l'Europe des Traités (1933-1940), dir. R. FABRE.

PRIN, Armand, Frère Camille de Jésus (1837-1915) de l'Institut des Frères des Écoles Chrétiennes, dir. M. LaunaY.

RouAud, Sébastien, L'action culturelle de la France au Japon dans l'entre-deuxguerres, dir. J. WEBER.

SARNAY, Jean, L'insurrection crétoise de 1897-1898, étape décisive vers l'annexion de la Grèce, dir. R. FABRE.

SAUVAGET, Aurélie, L'Action catholique ouvrière dans le diocèse de Nantes de 1950 à 1975, dir. M. LAUNAY.

ScHMIT, Gaëlle, Les intellectuels français et la guerre en ex-Yougoslavie, juin 1991décembre 1995, dir. F. Bozo.

\section{0}

\section{Histoire de l'art}

PEAU, Karen, Parures et bijoux mérovingiens des collections du Musée Dobrée à Nantes, dir. C. BECK.

\section{Archéologie}

CORNET, Céline, Usages historiques et environnement mental d'un château de marches du XI au XX siècle. La forteresse de Pouance (Maine-et-Loire), dir. J.-C. MEuRET.

LACOSTE, Nicolas, Les parures néolithiques au musée Dobrée. Classement, inventaire et amorce d'analyse, dir. J.-C. MEURET.

\section{Histoire ancienne}

BASSOMPIERRE, Karène, Le manoir de La Touche : historique et archéologie du bâti $X V^{e}$-XXe siècles, dir. J. PEYRAS.

FOUCHER, Christian, La guerre dans l'Histoire Auguste, dir. J. PEYRAS.

MEsPle, Pierre, Étude sur le commandement militaire des sources littéraires aux écrits d'Onasander, dir. J. PEYRAS.

\section{Histoire médiévale}

Joguet, Virginie, Les témoignages de l'expérience mongole, d'après les voyages de Guillaume de Rubrouck (1253-1255) et d'Ibn Battûta (1327, 1333-1335), dir. (M.) Clouzot.

LEFEBVRE, Arnaud, L'Évêché de Maillezais pendant la guerre de Cent Ans (13171455), dir. (J.-L.) SARRAZIN.

MAROLlEAU, Jean-Luc, L'abbaye de Maillezais dans les écrits des sociétés savantes au XIXe siècle, dir. (J.-L.) SARRAZIN.

QuATREBARBES, Ségolène de, Les relations entre les Ducs de Bretagne et les Rois de France de 1213 à 1488 vues par les chroniqueurs français, dir. (J.-L.) SARRAZIN.

TURPAUD, Baptiste, La seigneurie de La Jou de 1362 à 1515, dir. (J.-L.) SARRAZIN.

\section{Histoire moderne}

ALBERT, Raphaël, Louis-Antoine de Bertellet consul de France à Livourne (17631796), dir. J.-P. Bors.

AUDRAIN, Hervé, La Chambre des comptes de Bretagne au temps de la Fronde, dir. D. Le PAGE.

BATEAU, Caty, La politique de reconquête et de pacification de la Bretagne par Henri IV 1589-début XVII siècle, dir. D. LE PAGE. 
BEDREDE, Thomas, La présence française à Alep de 1749 à 1791, dir. C. HERMANN.

BILY, Stéphanie, Les officiers royaux de la Sénéchaussée de Guérande de 1700 à 1789 , dir. G. SAUPIN.

Bulteau, Séverine, Étude économique et sociale d'une famille de négociantsarmateurs nantais : les Grou 1698-1774, dir. G. SAUPIN.

COTTEN, Benoît, Le capital d'une exploitation agricole dans le pays de Fouesnant à la fin du XVIII siècle, dir. M. NASSIET.

DelaGe, Cédric, Les capitaines négriers à Nantes entre 1783 et 1793, dir. G. SAUPIN.

Denols, Perrine, Madame de Sévigné et la province, dir. D. LE PAGE.

DESPAS, Stéphanie, La façon d'habiter : six résidences nobles du Sud de la Bretagne XVII ${ }^{-}$XVIII $e^{e}$ siècles, dir. M. NASSIET.

DuboIs, Mathieu, Culture et société en Bretagne d'après les Lettres de rémission 1520-1522, dir. M. NASSIET.

DugAST, Romain, Essai pour la détermination d'une noblesse seconde en Bretagne au XVe siècle, dir. D. LE PAGE.

DuliEu, Vincent, La famille Gaudin aux Sables-d'Olonne au XVIII siècle, dir. G. SAUPIN.

GAR2IN, Mathilde, La présence française à Gênes : aspects et influence politique, dir. J.-P. BoIs.

GuERY, Sébastien, Tallemant des Réaux et l'absolutisme du premier Dix-septième siècle dans les Historiettes, dir. D. Le PAge.

JOALLAND, Yann, La ferme des octrois de la ville de Nantes de 1635 à 1729, dir. G. SAUPIN.

JouRDAn, Cécile, Les Deurbroucq, une famille de négociants nantais dans le commerce des îles au XVIII siècle, dir. E. NOEL.

LE MOAL, Laure, Les rapports sociaux en Bretagne au XVI siècle (1523-1525) d'après les lettres de rémission, dir. M. NASSIET.

LE Roux, Grégory, Les Européens sur les côtes africaines dans la première moitié du XVIII ${ }^{e}$ siècle, dir. G. SAUPIN.

LE TERTRE, Laurent, Le Consulat de Philadelphie et la question de SaintDomingue 1793-1803, dir. M. BELISSA.
LECOUTRE, Matthieu, Le maréchal-général de Villars, tacticien ou stratège?, dir. J.-P. BoIs.

Marchand, Cyrille, "Bonnie Prince Charlie » et l'insurrection jacobite de 1745, dir. J.-P. BoIs.

MENARD, Héloïse, Ascension politique et sociale de la famille de La Tullaye XVI XVII siècles, dir. D. LE PAGE.

NAEL, Vincent, Violence, société et mentalités en Bretagne d'après les Lettres de rémission 1533-1534, dir. M. NASSIET.

OIRY, Wanda, François de Pages : un aventurier au siècle des Lumières, dir. É. NOEL.

PARADIS, Laurent, Les droits seigneuriaux festifs et sportifs en Haute Bretagne du $X V^{e}$ au XVIII siècle.

PICHERIT, Damien, L'art de la guerre dans les Écrits de Lancelot Turpin de Crissé, dir. J.-P. BoIs.

PODLESNIK, John, Les pontonniers et le génie à Angers au XIX $X^{e}$ siècle (18701895), dir. J.-P. BoIs.

RABAUD, Magali, Étude de l'évolution de la fiscalité et du personnel financier en Bretagne de 1574, dir. D. LE PAGE.

RAIMBAUD, Élodie, Les étrangers en Bretagne d'après les lettres de naturalité $X V T^{e}-1661$, dir. D. LE PAGE.

RAVAlLLE, Ludwig, La violence en Bretagne au temps de la Ligue (1585-1598), dir. D. LE PAGE.

RINCE, Catherine, Religion et croyances dans le théâtre de Molière ou de la nécessité de croire à la maladie imaginaire, dir. D. LE PAGE.

SEBILO, Aurélia, L'habitat d'une élite à Guérande sous la période Moderne : étude de 7 manoirs, dir. M. NASSIET.

TEIXEIRA, Mario, Les relations internationales entre la France et le Portugal de 1820 à 1834, dir. C. HERMANn.

TEsSIER, Christelle, Pouvoir et ascension d'une famille de la noblesse nantaise : les Harouys, dir. D. LE PAGE.

\section{Histoire contemporaine}

AGHIAD, Malika, Les contrôleurs civils sous le Protectorat français au Maroc 19121956, dir. A. TIREFORT. 
BARdoux, Pierre, Le système politique libérien sous la présidence Tubman, 1944-1969, dir. A. TIREFORT.

BOHEAs, Julie, Histoire d'une maison d'éducation devenue un établissement d'enseignement secondaire féminin : l'institution Blanche de Castille tenue par les religieuses ursulines, de sa fondation à la signature des contrats d'association avec l'État 1806-1967, dir. M. LAUNAY.

BONNEAU, Hélène, Jayalalitha : de l'écran à la scène politique. Spectacle et politique à Madras (1965-1999), dir. J. WEBER.

BourgoIn, Philippe, François Mitterrand et la construction européenne, de son élection en mai 1981 à la signature de l'Acte unique européen en février 1986, dir. J. WEBER.

Bourreau, Yannez, La personnalité de Bokassa de 1966 à 1979 vue à travers le journal Le Monde, dir. A. TIREFORT.

BRusetTI, Olivia, Gabriel Deshayes et les Soeurs de l'Instruction chrétienne de Saint-Gildas-des-Bois, dir. M. LaUnAY.

CASTRIC, Jessica, Les réactions françaises aux événements de Tiananmen 15 avril4 juin 1989 : étude de presse, dir. J. WEBER.

COUELIER, Romain, Le modèle éducatif colonial néerlandais : exemple de la faculté Littéraire aux Indes néerlandaises, dir. J. WEBER.

DANET, Laurence, Les fondations d'écoles par les sœurs de l'instruction chrétienne de Saint-Gildas-des-Bois de 1882 à 1939, dir. M. LaunAY.

DROUET, Ludivine, La vie paroissiale à Saint-Étienne-de-Montluc, dir. M. LAUNAY.

Dubreuil, Estelle, L'Eglise et la construction européenne vues par le journal La Croix, de 1989 à 2000, dir. M. LAUNAY.

DuPONT, Olivier, Les relations franco-britanniques à la fin de la guerre froide (1985-début 1991), dir. F. Bozo.

DUPRE, Boris, Anarchistes et réprouvés en Loire-Inférieure de 1890 à 1906, dir. J.-C. MARTIN.
DURAND, Marina, La guerre des sexes. Violences sexuelles et Cour d'assises en Loire-Inférieure sous la monarchie de Juillet, dir. J.-C. MARTIN.

DURASSIER, Nathalie, L'appel à la Tunisie : ses contributions et ses réactions à l'effort de guerre 1914-1919, dir. J. WEBER.

Esnault, Matthieu, La diplomatie française face à la révolution jeune turque et aux mutations internes de la Turquie (1908-1912), dir. R. FABRE.

FouQueT, Stéphane, L'image de Bill Clinton en France (1992-2000), dir. F. Bozo.

FRASLIN, Valérie, La première guerre balkanique 1912-1913, dir. R. FABRE.

Fromentin, Nadia, Charles Brunellière, républicain et artisan du socialisme dans la cité nantaise (1879-1917), dir. C. PomeYrols.

GARANDEAU, Valéry, Les essais nucléaires indiens et pakistanais en mai 1998, dir. J. WEBER.

GARAUD, Christelle, La caisse d'Epargne dans une ville portuaire des origines aux années 1950 : l'exemple de SaintNazaire, dir. M. LAUNAY.

Grandhomme, Hélène, Le Petit Sénégalais, un journal dakarois. Juin 1913février 1918, dir. A. TIREFORT.

GRANGIENS, Guillaume, Le Parti communiste français et l'Afrique Noire du temps de l'Union française au lendemain des indépendances (1956-1963), dir. R. FABRE.

GUIBERT, Isabelle, La question macédonienne de la fin $d u X_{X I}{ }^{e}$ siècle à la première guerre balkanique, d'après les archives de l'ambassade de France à Constantinople (Annexes), dir. R. FABRE.

Gullou, Audrey, Éducation physique, pratiques sportives et politique coloniale au Sénégal (1900-1939), dir. A. TIREFORT.

HERMAN, Kathryn, Des émancipations parallèles? La femme éduquée et la presse à Dakar (Sénégal), de l'indépendance à l'application du Code de la famille (1960-1972), dir. A. TIREFORT.

JEAN, Claire-Marie, Les vocations nantaises de la société des missions étrangères : XIXe et XXe siècles, dir. M. LAUNAY. 
JOUBIER, Anthony, La lutte contre l'esclavage en Afrique noire après le congrès de Berlin (1884-1914), dir. J. WEBER.

LANDREVILLE, Cécile, La crise franco-siamoise de 1893. Étude des relations diplomatiques entre la France, la GrandeBretagne et le Siam, dir. J. WEBER.

Lavors, Matthieu, Le syndicalisme à l'arsenal d'Indret et aux forges de BasseIndre dans les années 1960, dir. R. FABRE.

Le Bihan, Pascal, La Corne de l'Afrique dans la tourmente 1977-1991, dir. A. TIREFORT.

LE CORRE, Ingrid, Littérature et colonisation. La perception française de la colonisation dans la littérature indo-chinoise de l'entre-deux-guerres, dir. J. WEBER.

LE GofF, Gaëlle, Histoire de la paroisse de Bouaye de 1802 à 1910, dir. M. LAUNAY.

Le Grand de La Chanonie, Catherine, Définition d'une politique indigène en Tunisie : Paul Cambon 1882-1886, dir. J. WEBER.

Le Poec, Mélinda, Les Nantais et le pèlerinage de Lourdes aux XIX et XX siècles, de 1872 à 1920, dir. M. LAUNAY.

LEA, Maëlle, Le regard du journal Le Temps sur la colonie de la Guyane française (1870-1900), dir. J. WEBER.

LOUAH-LE TELLIER, Michèle, La Commission Arc Atlantique : évolution dans ses ambitions, son fonctionnement et ses actions au cours de la présidence française (1989-1998), dir. J.-C. MARTIN.

LugAND, Marion, L'enseignement primaire élémentaire en Tunisie, de 1881 à 1906, dir. J. WEBER.

LuONG, Mmylène, La conquête du Tonkin vue d'après Le Temps et The Times (1881-1885), dir. J. WEBER.

Michelot, Gaëlle, La politique extérieure de Rajiv Gandhi (1985-1989), dir. J. WEBER.

Morel, Nicolas, L'Amérique de George Bush dans l'opinion publique française, dir. F. Bozo.

RENAUd, Stéphanie, Histoire d'un jumelage : Saint Stanislas de Nantes et le
Dillmann-Gymnasium de Stuttgart, dir. M. LAUNAY.

TISSEAU, Violaine, Entre ombre et lumière : métis et métissage à Madagascar, entre Européens et Malgaches de 1905 à 1960, dir. A. TIREFORT.

TRUCHOT, Pascale, La presse et l'opinion publique françaises face à la révolution russe de 1905, dir. R. FABRE.

\section{9}

\section{Histoire de l'art}

MILLET, Sébastien, Les premières implantations chrétiennes à Nantes et dans sa proche région des origines au $X^{e}$ siècle, dir. C. BECK.

\section{Archéologie}

BERNIGAUD, Nicolas, La forêt du Gâvre et sa périphérie de la Protohistoire au Moyen Âge, dir. J.-C. MEURET.

\section{Histoire ancienne}

ALBERT, Anne-Sophie, Les ex-voto figures des centres guérisseurs étrusques, dir. J.-R. JANNOT.

GuICHAOUA, Mickaël, Histoire biblique et histoire classique dans la Chronica Majora d'Isidore de Séville, dir. J. PEYRAS.

MATRAs-Desgre, Marie-Laetitia, La guerre de siège dans le monde grec et hellenisé à travers l'ouvre de Diodore de Sicile (de Denys l'ancien à Demetrios poliorcete), dir. J. PEYRAS.

RONDEAU, Isabelle, Étude de la géographie des informations dans les chroniques latines $d u$ ve siècle (Chronique de Cassiodore, Chronique du comte Marcellin cum additamento, Chronique de Marius d'Avenches), dir. J. PEYRAS.

TACET, Armelle, La circulation des informations à travers l'étude des correspondances de Jean Chrysotome, Theodoret de Cyr et Firmus de Césarée ve siècle, dir. J. PEYRAS.

THEBAUD, Sébastien, La villa gallo-romaine des Cléons (Haute-Goulaine, LoireAtlantique), dir. J. PEYRAS.

THOREAUX, Fabien, La piété grecque dans les sanctuaires étrusques, dir. J.-R. JANNOT. 


\section{Histoire médiévale}

Autrusseau, Julie, Voyage et voyageurs arabes du Magrib à l'Égypte du XII au $X V^{e}$ siècle : étude comparative de quelques récits, dir. C. MAZzoli-GuintaRd.

Begaudeau, Sandrine, Pelayo et l'idée de reconquête dans les chroniques ibériques des VIII et IXe siècles, dir. J. TOLAN.

BOyer, Nicolas, Polémique et missions chrétiennes anti-islamiques au XIII siècle à travers deux oeuvres de Ramon Marti, le de Seta Machometi et l'Explanatio simboli Apostolorum, dir. J. Tolan.

CLEON, Guylaine, L'administration du diocèse de Nantes (1206-1305), dir. J.L. SARRAZIN.

GRONDIN, Marina, La polémique anti-musulmane par Pedro Pascual, dir. J. TOLAN.

Gulluaud Nathalie, La commanderie de Sainte Gemme en Bas-Poitou du XIII au $X V^{e}$ siècles, dir. G. SAUPIN.

LECHAPPE, Laurence, La place des Juifs et des Musulmans dans les oeuvres légales d'Alphone X le Sage, dir. J. Tolan.

MENARDAIS, Laurence, L'image de Philippe Auguste et Richard Coeur de Lion dans les chroniques et récits de la Troisième Croisade, dir. J. Tolan.

Poussiere, Nicolas, Champgillon commanderie du Bas-Poitou du XIII au XV siècles, dir. J.-L. SARRAZIN.

\section{Histoire moderne}

AIllET, Anne-Sophie, L'apparence dans la noblesse à Nantes au XVIII siècle: une étude du vêtement, dir. M. NASSIET.

BALland, Anne, Littérature orale et noblesse bretonne : le cas du Parc de Locmaria, dir. M. NASSIET.

BERTON, Mathias, La reproduction familiale d'une catégorie sociale : les paysans propriétaires en Saintonge aux XVII et XVII' siècles.

Billaud, Lydie, L'Université de Nantes du $X V^{e}$ au XVII siècle, dir. M. NASSIET.

BRIAND, Marion, L'habitat rural dans le pays de Châteaubriant au XVIII siècle, dir. M. NASSIET.

ChAze, Sandra, Les négociants irlandais à Nantes à la fin du règne de Louis XIV: 1685-1715, dir. G. SAUPIN.
Chotard, Pierre, L'affaire Chalais (3 mai19 août 1626). Culture politique et raison d' État, dir. G. SAUPIN.

Dufournaud, Nicole, Comportements et relations sociales en Bretagne vers 1530, d'après les lettres de grâce royale, dir. M. NASSIET.

GaUTIER, Matthieu, La question d'Orient de 1833 à 1841 à travers les archives de l'ambassade de France à Saint Pétersbourg, dir. C. HERMAnN.

GLOTAIN, Christophe, La reproduction de l'oligarchie municipale nantaise (15651589), dir. G. SAUPIN.

HANNECART, Hélène, Culture et société en Bretagne d'après les lettres de rémission, 1525/1530, dir. M. NASSIET.

LauCoIn, Chrystelle, La naissance du trafic antillais (1638-1660), dir. G. SAUPIN.

LE Guen, Olivier, La Colombie de la première moitié du XIXe siècle vue par des voyageurs français, dir. C. HERMANN.

LE Joncour, Marine, Van der Meulen peintre de Louis XIV, dir. J.-P. BoIs.

Metaireau, Rachel, Anne de Bretagne visite son duché en 1505, dir. D. LE PAGE.

Michaud, Damien, Les Joubert du Collet, une famille négociante nantaise au XVIII $e^{e}$ siècle, dir. G. SAUPIN.

Michon, Bernard, Les négociants nantais et la pêche à la morue à Terre-Neuve dans la seconde moitié du XVIII siècle.

MiLCENT, Christelle, La Traite négrière nantaise de 1731 à 1739, dir. G. SAUPIN.

PERRAUD, Karine, L'armement maritime nantais en période de guerre : étude de la traite et de la course. Guerre de Succession d'Autriche (1744-1747). Guerre de Sept ans (1756-1762). Guerre d'Indépendance américaine (17791781), dir. G. SAUPIN.

Plantin, Claire, Les De Luynes : étude économique et sociale d'une famille d'armateurs nantais au XVIII ${ }^{e}$ siècle (1711-1788), dir. G. SAUPIN.

RIALLAND, Fabien, L'isolement français sur la scène internationale entre la crise des duchés de l'Elbe et le traité de Berlin (1864-1878) à travers les archives de l'ambassade à Saint-Pétersbourg, dir. C. HERMANN. 
SIM, Gérald, Le Consulat français de Charleston (1793-1835), dir. J.-P. BoIs.

\section{Histoire contemporaine}

AlLARD, Mathieu, L'évolution de la Tchécoslovaquie vue par les diplomates français de 1945 à 1953, dir. F. Bozo.

BAUDET, Claire, La création de l'État d'Israël à travers l'opinion des catholiques français avril 1945-mai 1948, dir. M. LAUNAY.

BEILleVert, Maryse, Les fondations d'écoles par la congrégation des sœurs de Saint-Gildas-des-Bois entre 1842 et 1882 , dir. M. LAUNAY.

BLIDON, Marianne, Écriture de la guerre, écriture de l'histoire : étude sur les Mémoires de guerre, dir. J.-C. MARTIN.

BODINIER, Didier, Approche de la justice coloniale en pays Bété 1904-1940 : traditions et sujétion, dir. A. TIREForT.

BoIsSEAu, Aurélie, Mary Wollstonecraft (1759-1797) et la Révolution française : L'engagement d'une féministe radicale anglaise et l'élaboration d'une mémoire sélective, dir. J.-C. MARTIN.

Bossard, Sébastien, Fachoda vu par Le Temps et The Times, dir. J. WeBER.

BREAUD, Yoann, La vie paroissiale de Pouzauges 1801-1905, dir. M. LAUNAY.

Coutelle, Flavien, Les conséquences de la présence militaire française en $\mathrm{Hel}$ vétie sous le mandat du ministre plenipotentiaire Reinhard (février 1800août 1801), dir. J.-C. MARTIN.

DAVIAU, Marielle, La guerre d'Espagne vue par le Populaire de Nantes (18 juillet 1936-1 ${ }^{\text {er }}$ avril 1939), dir. C. PomeYrols.

DEBORDE, Carole, La mémoire de la ville de Cluj. 1913-1999: étude des problèmes identitaires à travers les politiques officielles et la mémoire collective, dir. J.C. MARTIN.

DENIAUD, Grégory, L'armée et la marine japonaise : objectifs, stratégies et tactiques de l'Empire du Soleil Levant de 1868 à 1914, dir. J. WEBER.

Du BoIsGueHENeuc, Marie, Jean Bouchaud itinéraire d'un peintre officiel (18911977), dir. C. POMEYROLS.
Dufour, Isabelle, Les sœurs gardesmalades de la Sainte Famille de Grillaud, dir. M. LAUnAY.

ÉTENDARD, Éric, L'abbé Desgranges JeanMarie : un ecclésiastique parlementaire (1874-1958), dir. M. LAUNAY.

FORTIN, Marie, Enseignement supérieur et diffusion de la culture française en Tunisie (1881-1914), dir. J. WEBER.

Froger, Stéphanie, Un musée dans sa ville: le Musée des Beaux-Arts de Nantes (1801-1910), dir. C. POMEYRols.

GAROCHE, Brieux, Le Mouvement nationaliste chinois et la Seconde révolution, vus par la diplomatie française (mai 1919-avril 1927), dir. J. WEBER.

GAVALAND, Laurence, La présence américaine à Nantes et Saint-Nazaire de 19511967, dir. F. Bozo.

GERARD, Nathalie, La politique française au Gabon, Togo et Congo-Brazzaville en 1963-1964 vue par la presse, dir. A. TIREFORT.

GLEDEL, Olivier, Les Cent-Jours en LoireInférieure, dir. J.-C. MARTIN.

GUIBERT, Magalie, La population de Machecoul sous le Consulat et l'Empire, dir. J.-C. MARTIN.

HERVE, Ghislain, Leon Brunschvicg : un intellectuel nantais sous la République, dir. J.-C. MARTIN.

LAUREC, Guénolé, Les Musulmans du Tamil Nadu, à travers l'exemple de la communauté du district du North Arcot 18571998, dir. J. WEBER.

LEGEAY, Anne Isabelle, Le problème Sikh au Pendjab indien de 1980 à 1984, dir. J. WEBER.

LEMAITRE, Gwenaëlle, Les relations francopéruviennes à travers la correspondance politique et commerciale des agents français à Lima (1850-1880), dir. C. HERMANN.

LEROUX, Wilfried, Les relations du Népal avec ses deux grands voisins: l'Inde et la Chine de 1947 à 1965, dir. J. WEBER.

LOYER, Aurélia, L'Inde de Lal Bahadur Shastri à travers la presse française juin 1964-janvier 1966, dir. J. WEBER.

MAHE, Patricia, Vannes au fil de l'eau : le rétablissement des fontaines publiques (1854-1880), dir. M. LAUNAY. 
Marchand, Christophe, Dans les colonies de l'AOF de 1900 à 1960 : que deviennent les métis franco-indigènes?, dir. A. TIREFORT.

Marchand, Déborah, Gandhi et la communauté indienne en Afrique du Sud d'après le Times 1893-1914, dir. J. WEBER.

MARTIN, Frédéric, Le cartel des gauches à travers la presse nantaise : dimension nationale et dimension locale, dir. R. FABRE.

Messager, Céline, La vie quotidienne au travers de la Feuille Nantaise : janvier 1797-avril 1798, dir. J.-C. MARTIN.

METIER, Thierry, Vers l'établissement d'un protectorat sur le Grand Duché de Bade : résistances et compromis à l'impérialisme français en Allemagne (18001806), dir. J.-C. MARTIN.

Morillon, Lucie, La politique libanaise de la France des Accords Viénot à leur dénonciation unilatérale (1936-1939), dir. J. WeBER.

PERRAUD, Agnès, La mémoire des appelés de la guerre d'Algérie, dir. J.-C. MARTIN.

Piesse, Gabriel, Pondichery de 1954 à 1963, de la République française à la République indienne, histoire d'une transition, dir. J. WEBER.

RAFFEGEAU, Mickaëlle, La politique étrangère d'Indira Gandhi de 1972 à 1977, à travers la presse, dir. J. WEBER.

Rataud, Maud, L'évolution de la société dakaroise de 1900 à 1930, à travers les archives de Police, dir. A. TiREForT.

SAUZEAu, Grégory, La conquête des républiques Boers (octobre 1899-juin 1900) perçue par les quotidiens Le Temps et The Times, dir. J. WEBER.

Stanescou, Thomas, Le débat en France sur la « crise » des Euromissiles : 19771987, dir. F. Bozo.

Trottet, Sylvie, La conquête de Madagascar à travers le journal Le Temps à la fin $d u$ XIX $X^{e}$ siècle, dir. R. FABRE.

Violleau, Antoine, Periyar E. V. R. une conception révolutionnaire et rationaliste de la société tamoule, dir. J. WEBER.

\section{8}

\section{Archéologie}

BERnaCHe-Assollant, Nolwenn, Étude de l'industrie mésolithique sous le tumulus de Dissignac à Saint-Nazaire (LoireAtlantique), dir. J. L'HeLgouach.

BlANCHARD, Johann, L'occupation de Chênehutte-les-Tuffeaux et Gennes-sur-Loire de la fin de l'indépendance gauloise au début du Haut Moyen Âge, dir. J.C. MEuRET.

LEROUX, Pierre-Yves, Le matériel néolithique dans la zone des carrières près du Grand Menhir de la table des marchands à Locmariaquer, dir. J. L'HELGOUACH.

Windels, Yann, Mauves-sur-Loire et son environnement à l'époque galloromaine, dir. J.-C. MEURET.

\section{Histoire ancienne}

BILlon, Séverine, Le paganisme dans l'œuvre d'Athanase, dir. J. PEYRAS.

GASTE, Grégory, La tactique à Rome des origines à la guerre gauloise d'après Tite-Live, dir. J. PEYRAS.

GuilLon, Jérôme, Le musée de la Rochesur-Yon, dir. J. PEYRAS.

GuitTon, David, Les céramiques sigillées. Collections du musée Dobrée (Nantes, Loire Atlantique), dir. J. PEYRAS.

LEVILLAYER, Amaury, L'intervention des démons dans l'histoire humaine selon les Apologistes grecs des If et III siècles, dir. J. PEYRAS.

MEURQuin, Philippe, La tactique de l'armée romaine dans les stratagèmes de Frontin, dir. J. PEYRAS.

OGER, Magali, La religion de Julien d'après ses écrits, dir. J. PEYRAS.

Pichon, Julien, Recherche sur l'image du prêtre en Etrurie antique, dir. J.-R. JANNOT.

Rousseau, Nelly, Comparaison de l'histoire religieuse de l'humanité selon les auteurs chrétiens et païens latins $\mathrm{I}^{e r} \mathrm{av}$. J.-C.-IVe siècles après J.-C. Varton - Tartullien - Minucius Felix - Arnobe - Lactance, dir. J. INGLEBERT.

\section{Histoire médiévale}


BEVEn-Bunford, Jean-Benoît, Juifs et chrétiens d'Orient à travers la chronique d'Ibn Iyas, Badâ'i Al-Zuhûr fi Waqầ'i al-Duhûr, (872/928 H-1467/1522), dir. R. DURAND.

BonNaud, Romain, La famille médiévale dans l'historiographie britannique depuis 1950, dir. R. DURAND.

BRIAND, Julien, Un exemple d'exploitation de salines : les possessions des Blanchet dans la Baie de Bourgneuf à la fin du Moyen Âge, dir. M. LE MENE.

BRUNET, Gaëtan, La pratique de la guerre au VI siècle dans l'histoire des Francs de Grégoire de Tours, dir. R. DURAND.

BuRILlon, Thomas, Le défi de Dieu: le sentiment religieux dans la littérature médiévale en France, vers 1100-vers 1270, dir. R. DURAND.

Chaffin, Frédéric, La défense de la ville de Nantes au Xve siècle, dir. M. LE MENE.

Citron, Jean-Christophe, L'Écosse et les Écossais à la fin du Moyen Âge, dir. M. Le Mene.

FORET, Pauline, La place des chrétiens mozarabes dans la société hispanomusulmane du début du $X^{e}$ siècle au milieu du XII siècle, dir. J. TOLAN.

MotTe, Matthieu, Les Bretons vus par Froissart, dir. R. DURAND.

Peigne, Sébastien, Les évêques de Nantes de 1059 à 1213, dir. J.-L. SARRAZIN.

Rouxel, Arzhela, La place de Jérusalem dans l'idéologie de Saladin, dir. J. Tolan.

\section{Histoire moderne}

BERRY, Patrick, La nation française à Cadix de 1775 à 1791, dir. C. HERMAnN.

Bodiguel, Hervé, La présence française à Smyrne de 1774 à 1789, dir. C. HERMANN.

BouARIDJ, Stéphane, Historiographie de la bataille de Rossbach : de 1757 à nos jours, dir. J.-P. BoIs.

Cathelineau, Antoine, Le journal de bord de l'Hermione : campagne 1780-1782, dir. J.-P. BoIs.

FLEURANCE, Dimitri, Les officiers du présidial de Nantes au XVIII siècle, dir. G. SAUPIN.
Helbert, Stéphane, Doré du Perron, un négociant nantais dans la seconde moitié du XVIII siècle, dir. G. SAUPIN.

JANTON, Laurence, Culture et société en Bretagne d'après les lettres de rémission 1534-1574, dir. M. NASSIET.

PINEAU, Laure, Un négociant-armateur nantais : Louis Drouin 1722-1813: approche économique et sociale d'une vie liée à l'armement, dir. G. SAUPIN.

PINOT, Olivier, Le cadre de vie dans deux châteaux bretons aux XVII et XVIII siècles : permanences et changements, dir. M. NASSIET.

ROGER, Laurence, La noblesse de l'élection de Fontenay-le-Comte à la fin du XVII siècle et au début XVIII ${ }^{e}$ siècle, dir. M. NASSIET.

RoUCHON-BoRIE, Bérangère, Un notable nantais Panégyriste de sa ville : Enjeux et fondements. Étude de l'ouvrage de Jean Boutin de Chamballan et des Perrines, Apologie pour la communauté de Nantes et autres communautés contre la préséance prétendue par la communauté de Rennes à la tenue des Estats de la province, 1619.

ROUILLER, Carole, Culture et société d'après les procédures criminelles dans le marquisat de la Guerche (2e moitié du XVIII siècle), dir. M. NASSIET.

\section{Histoire contemporaine}

BEGUE, Sandrine, Le défi goanais dans la décolonisation. La bataille diplomatique indo-portugaise, dir. J. WEBER.

BERNARD, François, L'intégration d'une Caisse d'Epargne dans la ville : l'exemple de Nantes de 1914 à 1950, dir. M. LAUNAY.

Chaigneau, Karine, Les manifestations de l'idéologie vichyssoise dans l'enseignement à Nantes (1940-1944), dir. C. Pomeyrols.

ChATELLIER, Maxime, Les relations sinocommunautaires de 1975 à 1997, d'après des sources occidentales, dir. J. WEBER.

Chedorge, Cédric, La propagande du Reich et la minorité allemande de Roumanie 1919-1939: d'après les archives diplomatiques françaises, dir. T. BURON. 
Éon, Cécile, L'assistance médicale française en Chine de 1901 à 1937, dir. J. WEBER.

GALAUD, Christelle, Image d'un conflit : la Nouvelle-Calédonie à travers la presse quotidienne régionale (18 novembre 1984 au 30 septembre 1985), dir. J.-C. MARTIN.

GAUFFENY, Sandrine, Le père Prodhomme et la mission du Laos (1876-1920), dir. J. WEBER.

GiRARDEAU, Sophie, L'évolution d'une métairie vendéenne du XIX au XX siècle : La Chauvelière, dir. J.-C. MARTIN.

GlEmaIN, Denis, Le cinéma en Mayenne sous l'Occupation (1940-1944), dir. C. PomeYrols.

GuIHEux, Christelle, La politique culturelle de la France en Suisse pendant l'Entredeux-guerres, dir. Y.-H. NouallHat.

Harcouet, Céline, L'image de l'homme Noir à travers les Annales de la pro- pagation de la foi (1840-1900), dir. M. LAUNAY.

HOULBERT, Jérôme, Un évêque peu connu, Mgr Latieule, dir. M. LAUnAY.

Houp, Valérie, La paroisse d'Orvault 18031914, dir. M. LAUNAY.

JAUNET, Samuel, Bourgneufen-Retz sous la Première République. Aspects politiques et démographiques, dir. J.-C. MARTIN.

LALANDE, Nicolas, Le commandant Massoud vu par la presse française de 1978 à 1992, dir. J. WEBER.

Le Denmat, David, Saint-Louis du Sénégal de 1848 à 1854 : du comptoir à la ville coloniale, dir. A. TIREFORT.

LECLAIR, Franck, Deux banques françaises en Chine : la Banque de l'Indochine et la Banque Industrielle de Chine (18941937), dir. J. WEBER.

LOYER, Vincent, La guerre russo-japonaise (1904-1905) vue par la diplomatie française, dir. J. WEBER.

MATHE, Stéphane, Le général Turreau et les colonnes infernales, discours his- 
toriques, guerre civile et production de la vérité, dir. J.-C. MARTIN.

Morel, Aline, Les Français en Chine pendant la révolte des Boxeurs, 1898-1902, dir. J. WEBER.

PetrT, Émilie, Paul Griveaud dans la vie politique nantaise : un républicain dans la cité 1883-1909, dir. C. POMEYROLS.

POIRIER, Nicolas, Les baleiniers français en Nouvelle-Zélande, vecteurs des ambitions coloniales de la France (18351846), dir. J. WEBER.

POTIER, Christophe, La question de l'Anschluss de 1920 à 1929 vue par les diplomates français, dir. T. BURON.

POUPARD, Maud, L'historien Richard Cobb, 1917-1996, dir. J.-C. MARTIN.

PREAU, Anne, Le monastère de la visitation Sainte Marie de Nantes au XIX siècle, dir. M. LaUnAY.

RIVALAIN, Rose-Line, L'affaire de l'enlèvement des sept moines de Tibhirine et son traitement par la presse française, dir. M. LaUnAY.

Robin, Rachel, Mémoire des Sables d'Olonne et de la Chaume, dir. J.-C. Martin, dir. J.-C. MARTIN.

Robin, Thomas, E. P. Thompson, 1924 1993 : l'historien dissident, dir. J.-C. MARTIN.

ROCHETEAU, Julien, La décolonisation des Indes Néerlandaises vue par les diplomates français 1945-1949, dir. J. WEBER.

Theval, Vincent, Les Cahiers du cinéma en 1968. Politique, critique et cinéma, dir. C. PomeYrols.

TUTUNDJIAN, Guillaume, La Colombie Britannique vue par les Consuls de France, à l'aube du XXe siècle (1891-1911), dir. Y.-H. NOUAILHAT.

YRIGOYEn, Hubert de, L'Université de Nantes de 1969 à nos jours, dir. Y.-H. NOUAILHAT. 


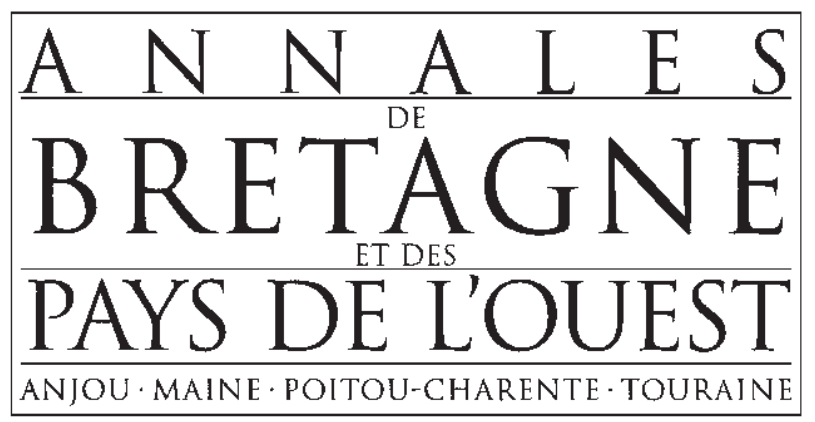

\section{ABONNEMENT}

France, 4 numéros : $32 €$-ÉTRANGER, 4 numéros : $40 €$ (Franco de port)

ADRESSE D'EXPEDITION

(pour toute commande)

Nom

Prénom

Organisme

Adresse

Code postal

Ville

Pays

Ce bon de commande accompagné d'un chèque à l'ordre de Agent comptable de l'Université Rennes 2 est à envoyer à l'adresse suivante :

Presses Universitaires De RenNes

Campus de La Harpe

2 rue du Doyen-Denis-Leroy

35044 RENNES Cedex

FRANCE 


\section{QUELQUES REGLES DE PRESENTATION DES TEXTES A L'USAGE DES AUTEURS}

1 - Indiquer sous le titre de l'article la qualité et l'adresse institutionnelle de l'auteur Ex. : Xxxx Xxxxxx, maître de conférences en histoire contemporaine, université de X., groupe de recherches de rattachement.

2 - Remplacer le plus possible les majuscules (sauf pour les lettres initiales des noms propres) par des petites capitales. Ceci dans les titres, les sigles, les noms d'auteurs... Indiquer aussi les siècles en petites capitales : $\mathrm{XV}^{\mathrm{e}}$ siècle et non XVe siècle.

Accentuer les majuscules.

3 - Abréviations : en user le moins possible

Pas d'abréviations pour le nom des revues, surtout pour les revues locales qui ne sont connues que des locaux.

Pour les dépôts d'archives :

- ne pas mettre ADIV ou AD 35 mais : Arch. dép. d'Ille-et-Vilaine, du Morbihan...;

- préférer Arch. nat. à AN;

- mettre les noms des bibliothèques en entier.

Développer les sigles entre parenthèses lors de leur première occurrence.

\section{4 - Références bibliographiques}

- mettre toujours et partout les prénoms et les noms (ne pas abréger le prénom par une initiale);

- utiliser les petites capitales pour le nom propre et non les majuscules, utiliser les minuscules pour le prénom; les séparer par des virgules.

Nom, Prénom, Titre de l'ouvrage en italique, Lieu d'édition, éditeur, " collection " (éventuellement), année, pages.

Une bibliographie peut être donnée en fin d'article si cela est nécessaire.

\section{5 - Forme des notes}

Préférer la forme traditionnelle (référence biblio. développée lors de sa première occurrence, abrégée ensuite) à la forme anglo-saxonne (nom, date, page).

\section{6 - Titres et sous-titres}

Il est préférable de s'en tenir à deux niveaux. Mais si cela s'avère nécessaire afin de ne pas obtenir de trop longues pages trop compactes, un troisième niveau d'intertitres peut être utilisé.

\section{7 - Cartes et documents photographiques}

- Les cartes au trait devront être d'une EXCELLENTE QUALITE GRAPHIQUE sans collage hasardeux ni légende manuscrite.

- Il est toujours préférable de FOURNIR LES ORIGINAUX, sinon, un fichier informatique au format .EPS pour les cartes et plans ou .TIF pour les photographies. en aucun cas les images seront intégrées au fichier word seulement : IL FAUT EGALEMENT LE FICHIER SOURCE.

8 - Autres questions non évoquées ici...

consulter la rédaction à l'adresse ci-dessous.

9 - Donnez une adresse mail et téléphone où l'on puisse vous joindre facilement au moment où l'on fait la mise en page de votre article.

\section{Contact}

Annie Antolne, Secrétaire de l'Association pour la publication des $A B P O$ université de Rennes 2, Département Histoire, Place du Doyen-Henri-Le-Moal - 35043 RenNes Cedex e-mail : annie.antoine@uhb.fr

Les manuscrits sont à envoyer à l'adresse ci-dessus

Ouvrage achevé d'imprimer

sur les presses du service reprographique

de l'Université Rennes 2 Haute-Bretagne

en juin 2006

Imprimé en France 